UCR L-53266

Distribution Category-11

\title{
An Analytic Model for Surface Ground Motion With Spall Induced by Underground Nuclear Tests
}

\author{
Donald H. MacQueen
}

Manuscript Date: April 1982

UCRL -53266

DE82 016783

LAWRENCE LIVERMORE LABORATORY University of California $\bullet$ Livermore, California 94550 


\section{Contents}

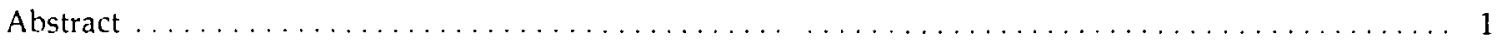

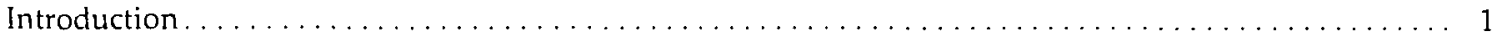

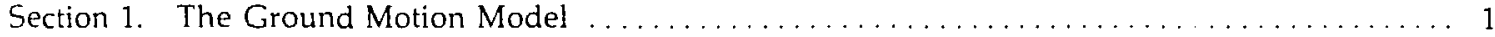

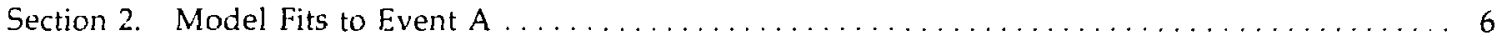

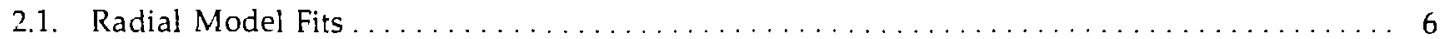

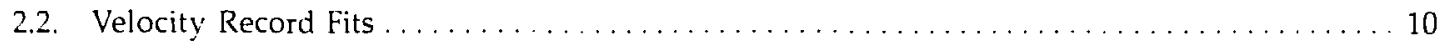

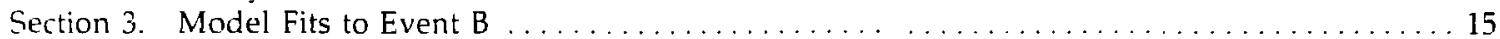

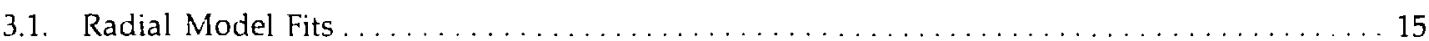

3.2. Velocity Record Fits ... . . . . . . . . . . . . . . . . . . . . . . . . . . . . . 19

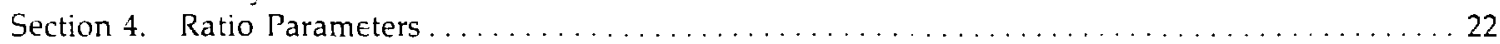

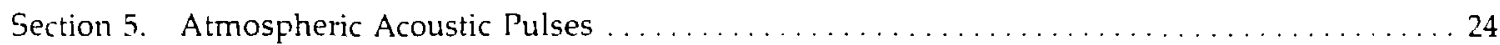

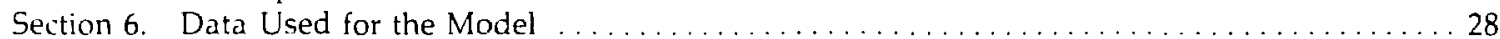

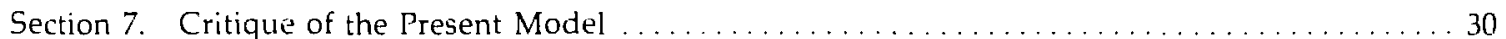

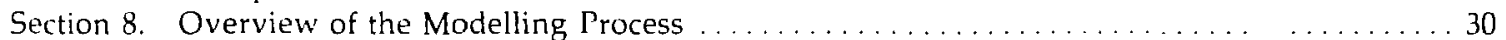

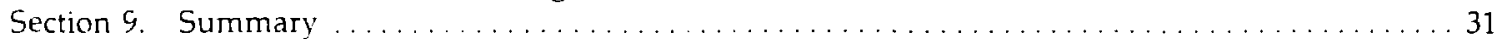

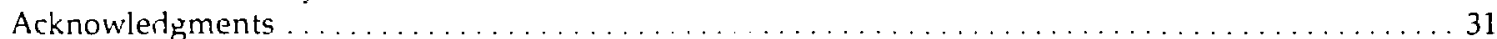

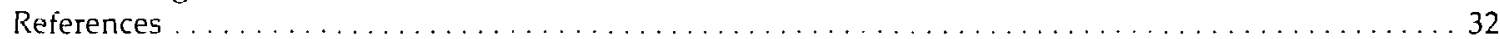

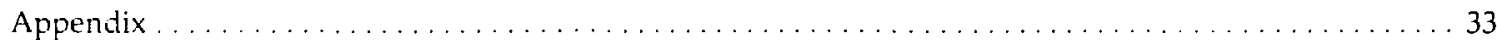




\title{
An Analytic Model for Surface Ground Motion With Spall Induced by Underground Nuclear Tests
}

\begin{abstract}
This report provides a detailed presentation and critique of a model used to characterize the surfici ground motion following a contained, spalling underground nuclear explosion intended for calculation of the resulting atmospheric acoustic pulse. Some examples of its use are included. Some discussion of the general approach of ground motion model parameter (xtraction, not dependent on the specific model, is also presented.
\end{abstract}

\section{Introduction}

The surfacr ground motion model discussed here was developed by S. I. Warshaw of Lawrence livermore National Laboratory (LLNL) for use in calculations of the atmospheric disturbance following an underground nuclear test. The procedure described in this seport for applying the model was developed by the author. The primary focus of the discussion is the surface ground motion. However, a few examples of the atmospheric disturbance calculations are included. The model is specifically limited to events in which spall occurs, hut it could be adapted for non-spalled events.

\section{Section 1: The Ground Motion Model}

During an underground nuclear test (UGT) an array of surface accelerometers is placed at various distances and directions from surface ground zero (SGZ). The recorded acceleration time histories, together with the velocity histories (usually derived by integration but sometimes measured directly), form a typical ground motion data set. Ground motion stations further away from SGZ generally experience accelerations of lesser magnitude, and ground motion stations at different azimuths often record substantially different acceleration histories, even when the stations are at the same distance from SGZ. Therefore, a fully comprehensive ground motion model would have to take into account the complexities of geology and terrain variations. The model described here is relatively simple and assumes that (a) the medium through which the shock wave passes is homogeneous, (b) the ground surface is flat and horizontal, and $(c)$ the ground motion is cylindrically symmetrical. In addition, our model is specifically intended to treat spall, since it occurs quite frequently in contained UGTs.

Our basic model is that the surface acceleration experienced at a given distance from SGZ after a device is exploded is a piecewise linear function of time. The integral of this acceleration function is a velocity function; both of these functions are shown in Fig. 1. Each of the 11 vertices, which occur at times $t_{1}$ through $t_{11}$ on the acceleration curve, and each of the four velocity extrema labeled $v_{1,}, v_{1,}, v_{\gamma}$, and $v_{i}$ is provided with a mathematical model of some form. In addition, the time intervals $t_{5}-t_{4}$ and $t_{7}-t_{6}$, have models. (Hereafter, $t_{5}-t_{4}$ is denoted $J t_{\text {.) }}$ The interval from $t_{5}$ to $t_{6}$ is the period of ballistic free-fall characteristic of UGT spallation. It will normally have an acceleration of $-\mathrm{g}$. The acceleration spikes at $t_{2}$ and $t_{-}$are due to the initial blast wave and the subsequent slapdown of the spalled layer, respectively.

The specific mathematical models for the various points on the acceleration and velocity curves fall into three classes:

i. A function of distance from SGZ.

2. A constant proportion of the acceleration of gravity.

3. A constant ratio between values at two points. 

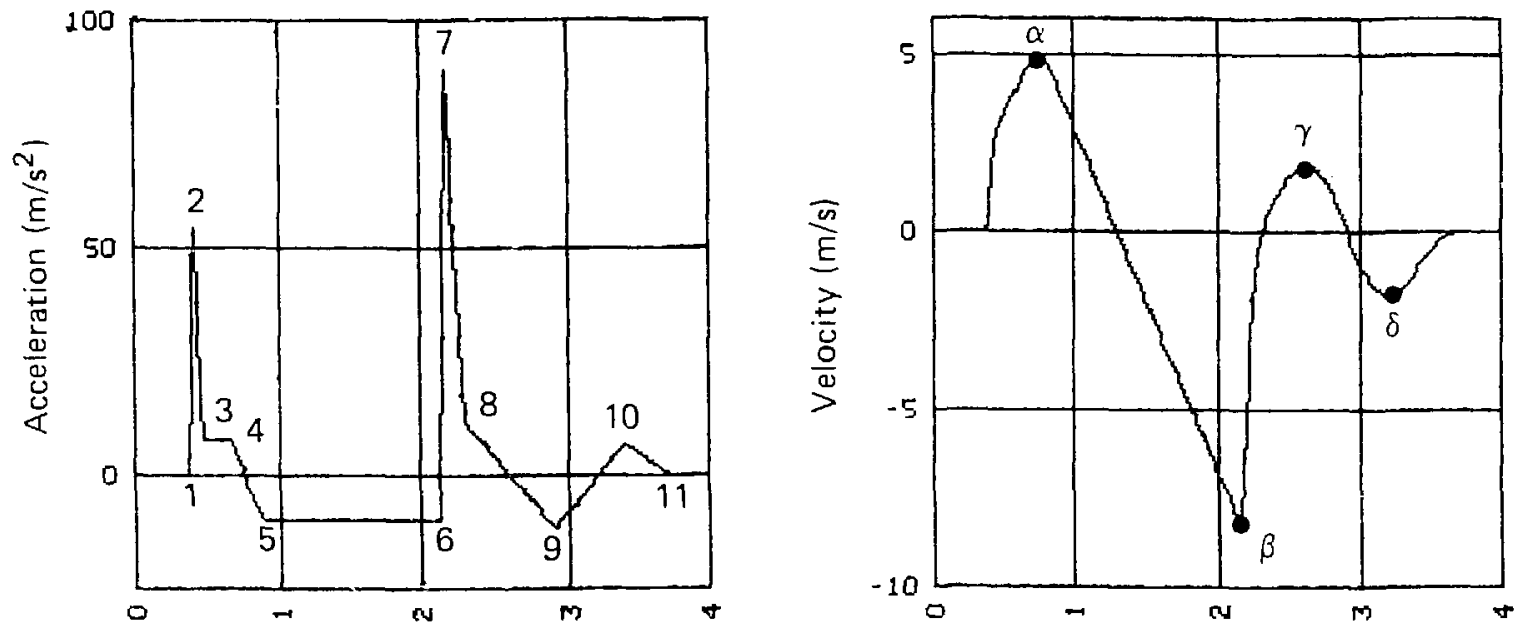

Time (s)

Figure 1. The 10-segment piecewise linear acceleration time history model and its associated piecewise quadratic velocity time history.

The acceleration and velocity extrema $a_{2}, a_{7}, v_{11}$, and $v_{1}$ and the time interval $د$ are in the first class. We have used two different radial functions for these points. The first, developed by Warshaw, is of the form

$y=\frac{A}{1+\left(B \frac{R C}{Z B}\right)^{\prime}}$,

where $R C$ is the surface distance from $S G Z, Z B$ is the explosive placement depth, and $A, B$, and $C$ are parameters to be estimated. Each of the above five quantities using this radia: function (whicn we will refer to as the Warshaw model) is allowed to have separate parameter values.

The second function is the generally accepted ${ }^{l}$ power law for $a_{2}$ and $v_{11}$. It is of the form

$y=K\left(\frac{V R C^{2}+Z B^{2}}{Y^{1 / 3}}\right)^{-K}$

where $Y$ is proportional to the energy released in the explosion, and $K$ and $E$ are the parameters to be estimated. We refer to this model as the Bernreuter model because we first learned of it through Bernreuter, Jackson, and Miller, ${ }^{2}$ who used it for determining how to protect delicate diagnostic instrumentation against violent UGT induced surface motions.

Table 1 gives the variable names for each of the radial parameters we have been using in our codes. Some examples of the range of shapes these functions can take are given in Figs. 2 and 3 . For the purposes of comparison all are scaled to tike on a maximum value of 1.0 .

The Bernreuter model applies to the initial velocity and acceleration peaks, but it does not appear to have been used for any subsequent extrema. Given that one has decided to use this model for $v_{11}$ or $a_{2}$, there are two possibilities for $v_{b j}$ and $a_{7}$, both of which we have used. The first is to use the Warshaw model for $v_{i f}$ and $a_{7}$, while the second is to assume a linear relaiionship between $a_{2}$ and $a_{7}$ and hetween $v_{a}$ and $v_{1 j}$. That is, $a_{7}=F_{2}$ or $v_{b}=F v_{a r^{\prime}}$ where the parameter $F$ is to be estimated by linear regression. In the latter case the radial dependence of $a_{7}$, has the same shape as that of $a_{2}$, differing only by a scale factor, and similarly for $v_{13}$ and $v_{11}$. In the first case $a_{7}$ and $a_{2}$ will generally drop off at different rates at different 
Table 1. Radial parameters of the first class. These parameters are used to determine the values of the points of the model that have a radial dependence. (See the appendix for details.)

\begin{tabular}{|c|c|c|c|c|c|c|}
\hline \multirow[b]{3}{*}{ Point } & \multicolumn{6}{|c|}{ Model } \\
\hline & \multicolumn{3}{|c|}{ Warshaw } & \multicolumn{3}{|c|}{ Bernreuter } \\
\hline & $\overline{\mathbf{A}}$ & $\bar{B}$ & $\mathrm{C}$ & $\mathrm{K}$ & $\overline{\mathbf{E}}$ & $\mathbf{F}$ \\
\hline$a_{2}$ & APOS & $\mathrm{FA}$ & XPA & & & \\
\hline$a_{2}\left(Y^{1 / 3}\right)$ & & & & ACON & AEXP & \\
\hline a- & ANEG & FB & XPB & & & \\
\hline$a_{-}\left(Y^{1: 3}\right)$ & & & & $A C O N$ & AEXP & ARAT \\
\hline $\mathbf{v}_{u}$ & VPOS & FV & XPV & VCON & VEXP & \\
\hline $\mathbf{v}_{. j}$ & VNEG & FW & XPW & VCON & VEXP & VRAT \\
\hline$t_{5}-t_{4}$ & DTSH & FT & $X P T$ & & & \\
\hline
\end{tabular}

distances from $S G Z$. The parameters $K, E$, and $F$ of the Bernreuter model will have different values for $a_{2}$ and $a-$ than for $v_{11}$ and $v_{i j}$.

Given any set of parameters for the Bernreuter model it is easy to find a set of parameters for the Warshaw model which will match this fit to the data. In practice this is what we do when we decide to use the Bernreuter model for a particular set of data.

The point $v_{3}$ has a model which combines a radial dependence with a constant ratio. Explicitly, this is $\left.r_{:}=v_{1 .} \mid 1.0-(1.0-\mathrm{F} 8)\left[1+\left(\mathrm{FT} \frac{\mathrm{RC}}{\mathrm{ZB}}\right)\right]{ }^{\prime}\right\}$.

Note that when $R C=0$, the model reduces to $v_{3}=(F 8) v_{1 .}$.

Except for $\mathrm{a}_{11}$, the remaining points on the acceleration curve are all members of the second class of models: their magnitudes are a proportion of the acceleration of gravity (g) that does not depend on the distance from SGZ. In some cases the constant of proportion is a parameter to be estimated, and in other cases it is assumed by the model to be 0 . Table 2 gives the details. For example, the model says that $a_{1}=$ (F1)g and $a_{3}=(-r i) g$.

The remaining velocity extrema $v_{7}$ and $v_{i}, a_{10}$, and the time interval $t_{7}-t_{6}$ have the third type of modei, shown in Table 3 . The model says, for example, that $v_{3}=(F 4) v_{n}$. All remaining time and velocity points are determined using simpls constant acceleration calculations relating times, accelerations, and velocities. Formulas for these are given in the appendix.

Finally, the model must specify a surface arrival time for the seismic shock wave. For this, the speed of sound in the earth (VB) is estimated from the data using the following relationship:

$t_{2}=\frac{\sqrt{R C^{2}+Z B^{2}}}{V B}$

This estimate corresponds to the time at which a uniformly expanding spherical blast wave first reaches the flat surface of the ground.

A complete set of ratio parameters $\mathrm{F} 1$ through $\mathrm{F} 8$ and an appropriate set of radial parameters (Warsh.aw model for $\Delta t$ and Warshaw or Bernreuter parameters for the extrema), together with ZB and VB, are sufficient to specify the entire array of points $t_{1}$ tc $t_{11}, a_{1}$ to $a_{11}, v_{1}$ to $v_{11}, v_{1,}, v_{13}, v_{\gamma}$, and $v_{h}$ for any RC. The model includes an additional parameter that is essential for calculating the atmospheric acoustic Fulse. This is RCMAX, the radius beyond which no significant surface ground motion contribution to the acoustic pulse is assumed to occur. For the purpose of determining RCMAX, we assume that this is also close to the end of the spall zone. 


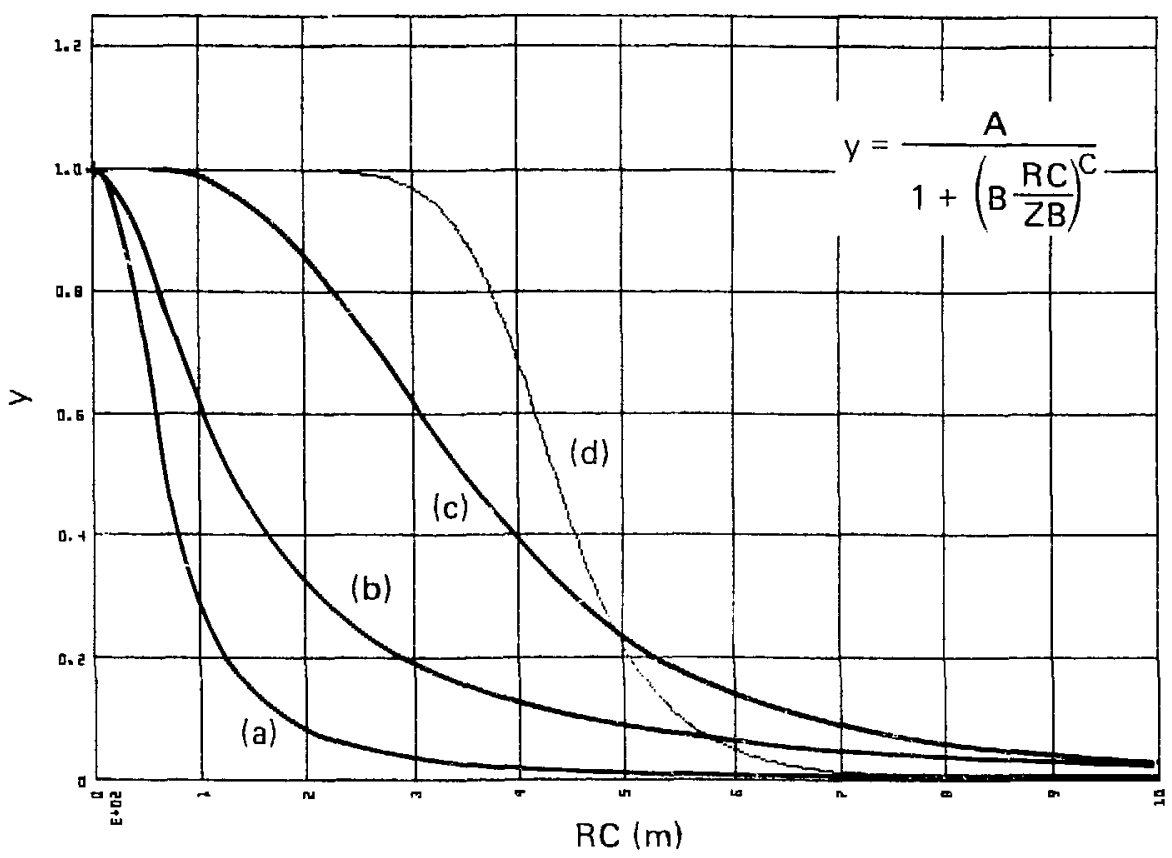

Parameters

(a)

$A=1.00$

$B=1.50$

$\mathrm{C}=2.25$

(b)

$A=1.00$

$\mathrm{B}=0.76$

$C=1.75$

(c)

$A=1.00$

$\mathrm{B}=0.29$

$\mathrm{C}=3.27$

(d)

$A=1.00$

$B=0.23$

$\mathrm{C}=9.28$

Figure 2. The Warshaw radial function. Four sets of parameters show the range of possible shapes the function can have. $Z B=100$ and $R C=$ the distance (in $\mathrm{m}$ ) from SGZ.

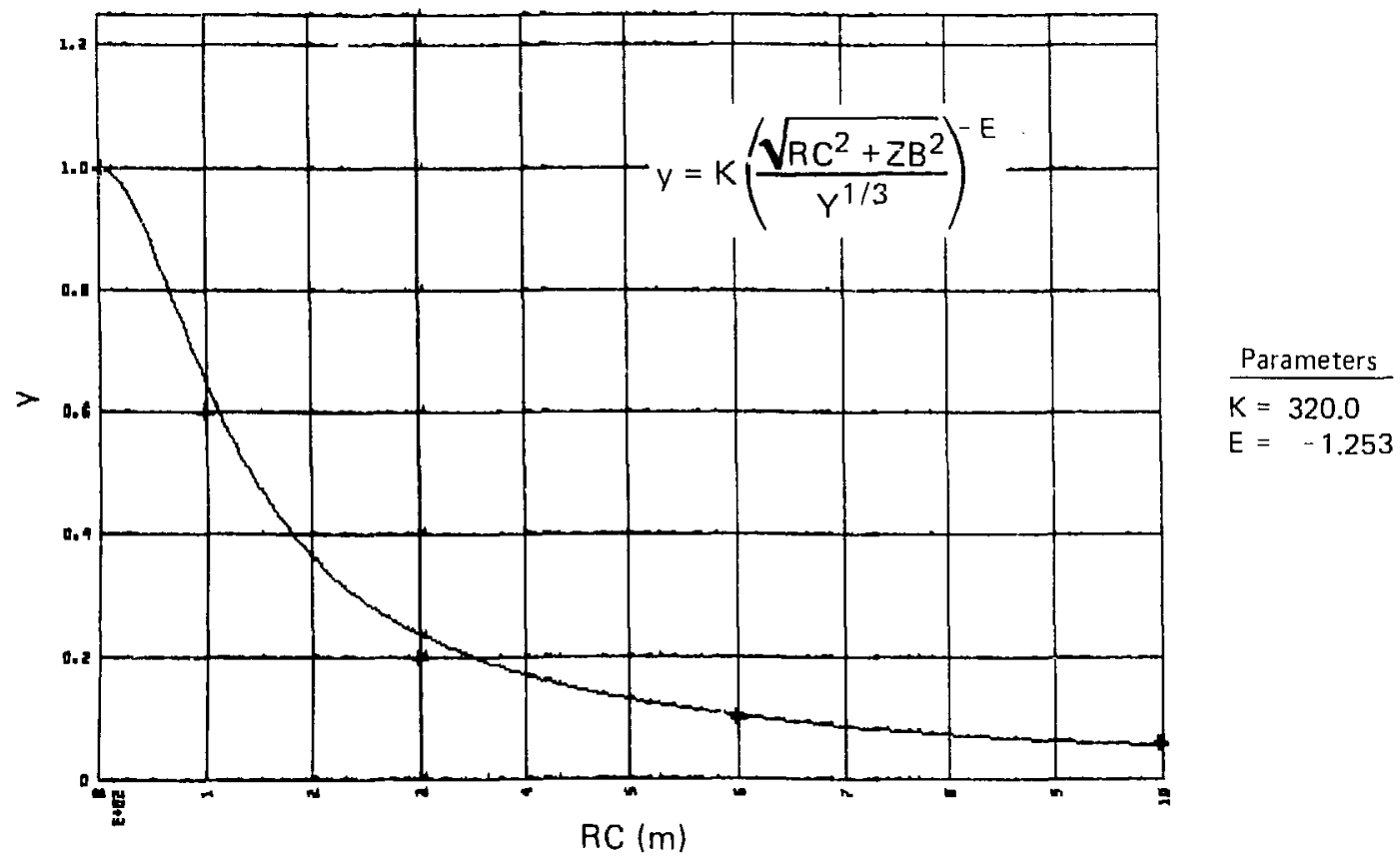

Figure 3. The Bernreuter radial function. One set of parameters shows the typical shape. $\mathrm{RC}=$ the distance (in $\mathrm{m}$ ) from SGZ. $Y=1.0$ and $Z B=100.0$. 
Table 2. Ratio parameters of the second class. These parameters are used to determine the value of the points of the model that are not radially dependent.

\begin{tabular}{ccc}
\hline Data point & Parameter & Constant \\
\hline$a_{1}$ & & 0.0 \\
$a_{3}$ & $F 1$ & \\
$a_{4}$ & $F 1$ & \\
$a_{7}$ & $-F 7$ & \\
$a_{6}$ & $-F 7$ & \\
$a_{4}$ & $F 2$ & \\
$a_{4}$ & $-F 2$ & \\
$a_{11}$ & & 0.0 \\
\hline
\end{tabular}

Table 3. Ratio parameters of the third class. These parameters specify a constant ratio between certain points on the model.

\begin{tabular}{cc}
\hline Ratio of data points & Parameter \\
\hline$a_{10} / a_{8}$ & $F 3$ \\
$v_{7} / v_{a r}$ & $F 4$ \\
$v_{d} / v_{3}$ & $-F 5$ \\
$\left(t_{-}-t_{6}\right) /\left(t_{2}-t_{1}\right)$ & $F 6$ \\
$v_{3} / v_{a r}$ & $F^{a^{a}}$ \\
\hline
\end{tabular}

${ }^{2} T$ his is only true at $R C=0$.

Before presenting some applications of this model, it will be useful to make some general remarks about the fitting procedure.

The Warshaw radial model is nonlinear in the parameters and is therefore fit using a nonlinear least squares routine. ${ }^{3}$ The Bernreuter model is linear in the logarithms of the variables and can be fit using ordinary linear regression. The primary distinction between these procedures is that the Bernreuter model minimizes relative errors, whereas the Warshaw model minimizes absolute errors. This is most important at large radii, where the data values are small. In such a case an error that would be sufficiently small in absolute value if it were near SGZ may be excessively large in relative value. For this reason the Bernreuter model tends to provide hetter fits at large radii.

We specify RCMAX to ensure that the ground motion beyond RCMAX will be considered negligible. This in turn indicates that the ratial fits to the data should have a small value at RCMAX. This does not always happen so in some cases a constraint must be imposed during the fitting calculations.

In order to implement the constraint, a value for RCMAX and an appropriately small value for the fit at RCMAX must be chosen. The latter is provided by Bernreuter et al., who state that the spall zone will normally extend to about where the peak particle velocity $\left(v_{10}\right)$ has fallen to about $2 \mathrm{ft} / \mathrm{s}$, or $0.6 \mathrm{~m} / \mathrm{s}$. In some cases data have been collected at locations beyond the extent of spall (indicated by lack of free-fall on the acceleration record). Then RCMAX can be estimated by interpolation between a data point within the spall zone and another outside it. In the not uncommon absence of such data there can be considerable difficulty; extrapolating data within the spall zone to a distance that will decrease the velocity to $0.6 \mathrm{~m} / \mathrm{s}$ often results in excessively large values of RCMAX.

An alternative, given by Eisler and Chilton, ${ }^{4}$ is to look at the time difference $t_{6}-t_{1}$. They find that this value decreases with radius to a certain point, becoming constant thereafter. They suggest that this point is the limit of spallation and so is a suitable approximation to RCMAX. This method requires more extensive accelerometer coverage than is usually available.

One important statistical aspect of the radial data should be mentioned. With most methods of data fitting, the analyst commonly assumes that the data have equal variances. This is most definitely not the case in this situation. Close to SCZ one would expect to find little variation in the medium through which the explosive shock wave passes, and so we expect the data to have small variances. In the middle ranges, the shock wave can pass on a slant through radically different ground materials to reach accelerometers at different azimuths. Hence, a larger variance is expected due to variations in gr ology and multiple reflections in different strata. At large radii the magnitudes are small, so the variances must necessarily be small. In terms of fitting, this means we should expect good fits close to SGZ and at large radii but be tolerant of relatively large errors at the middle distances.

The ratio parameters are usually obtained in two steps. The first takes the geometric mean of the available data ratios. This may produce good results, but often does not, especially for the parameters for points after slapdown. Second, the parameters are adjusted as necessary to improve the overall velocity record fits, and sometimes to make the model acceleration and velocity records look reasonable. By looking reasonable, we mean that the overall magnitudes and durations of the third and fourth velocity peaks are somewhat smaller than the first and second. This will be discussed in more detail in Section 4. 


\section{Section 2. Model Fits to Event A}

Here we present the fit of our model to the first of two sets of data. The first, called Event $A$, is an example of a relatively poor fit. The second, Event B, shows an excellent fit and will be discussed in Section 3.

\subsection{Radial Model Fits}

Figures 4- 8 present the Warshaw and Bernreuter fits for the radial parameters for Event $A$. The data at a radius of $15 \mathrm{~m}$ will be referred to as being at SGZ. The Bernreuter fits were obtained using the value $Y=1.0$.

The radial fits for the initial $\left(a_{2}\right)$ and slapdown $\left(a_{7}\right)$ acceleration peaks (Figs. 4-5) illustrate some of the problems that we often encountered.

The scatter of the data for $a_{2}$ is such that no real decrease in amplitude in the first $1000 \mathrm{~m}$ from SGZ is apparent. The fit reflects this fact and suggests a ground motion more like a piston than a flexible diaphragm. Thero is some possibility of rejecting the two extreme values $\left(85 \mathrm{~m} / \mathrm{s}^{2}\right.$ at $836 \mathrm{~m}$ and $22 \mathrm{~m} / \mathrm{s}^{2}$ at $707 \mathrm{~m}$ ), but the raw data records do not justify this, nor does doing so significantly affect the fit. Ti.e value for APOS, 47.40, is very close to the mean of the 12 points having a radius less than $1500 \mathrm{~m}$. This is appropriate, hecause the mean is the least squares point estimate of a set of data when other variables (in this case $R C$ ) are irrelevant.

The Bernreuter fit for $a_{2}$ (Fig. 4) has a nice shape and goes through the data fairly nicely if the extreme value at $836 \mathrm{~m}$ is ignored. However, it overestimates the acceleration at SGZ rather badly, and its sum of squared residuals is almost twice that of the Warshaw fit.

The measured data for $a_{z}$ (Fig. 5) is disturbing in several respects. Many of the data records show clipping of the slapdown acceleration peak. One such is the record at a radius of $1131 \mathrm{~m}$; like several

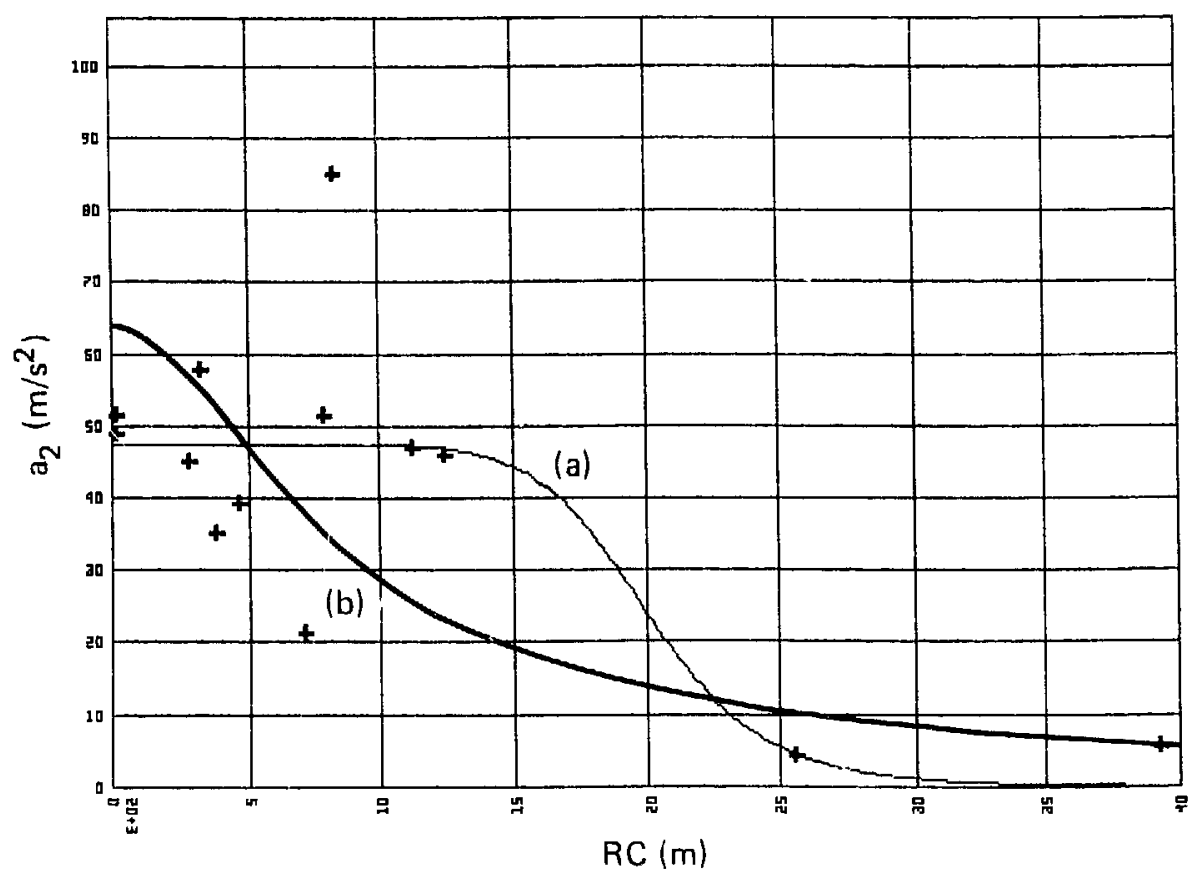

Parameters

(a)

APOS $=47.40$

$F A=0.32$

$\mathrm{XPA}=9.24$

(b)

$A C O N=2.61 E+05$

AEXP $=1.29$

ARAT $=3.75$

Figure 4. Comparison of the (a) Warshaw and (b) Bern reuter radial model fits to the initial acceleration peak $a_{2}$ for Event $A . Z B=640.0$ and $Y=1.0$. The sum of squared residuals for curve (a) is 2572.9 and for curve (b) 5109.1. 


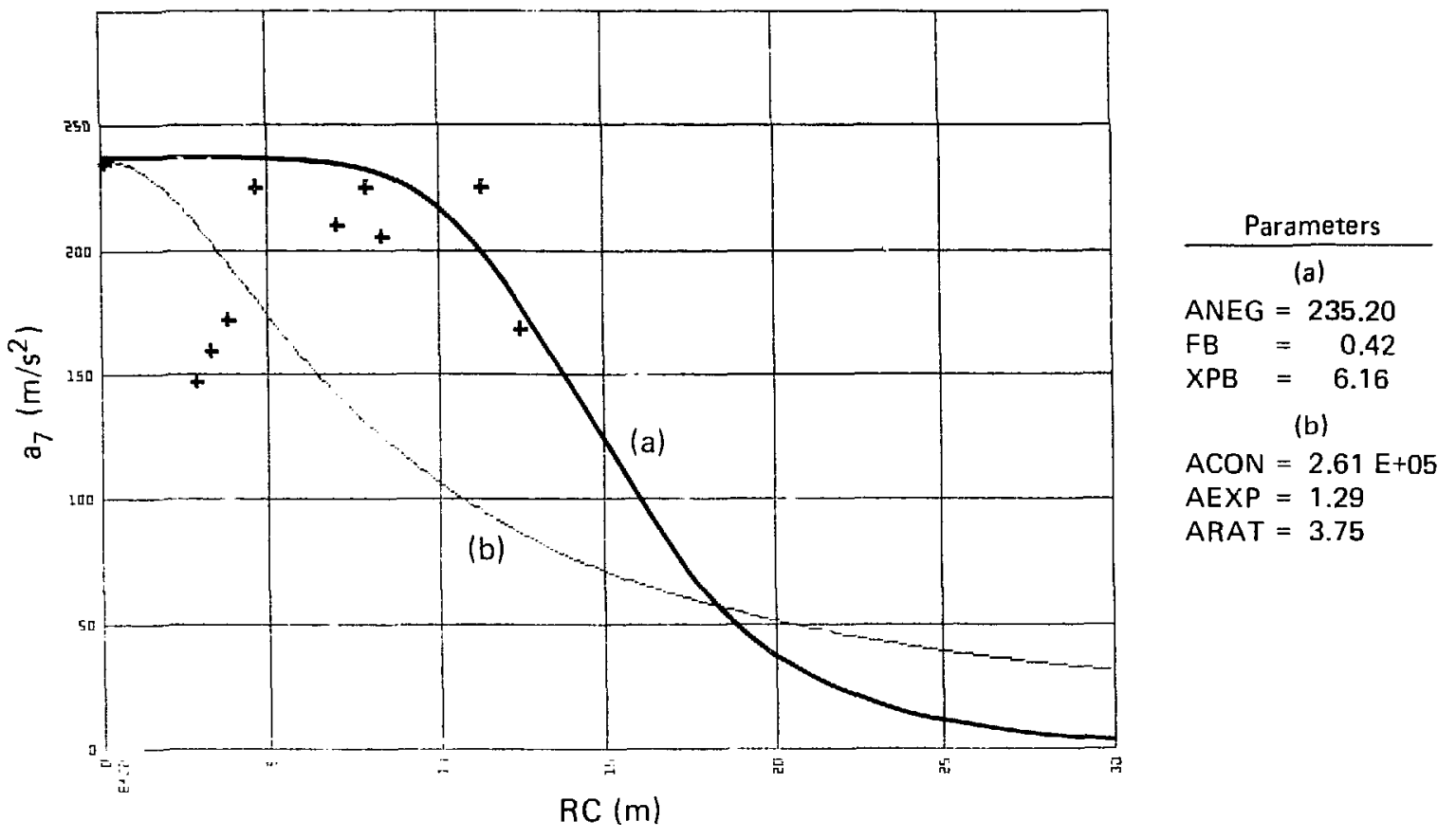

Figure 5. Comparison of the (a) Warshaw and (b) Bernreuter radial model fits to the slapdown acceleration peak $a_{7}$ for Event $A . Z B=640.0$ and $Y=1.0$. The sum of squared residuals for curve (a) is 375.3 .0 and for curve (b) 52894.0.

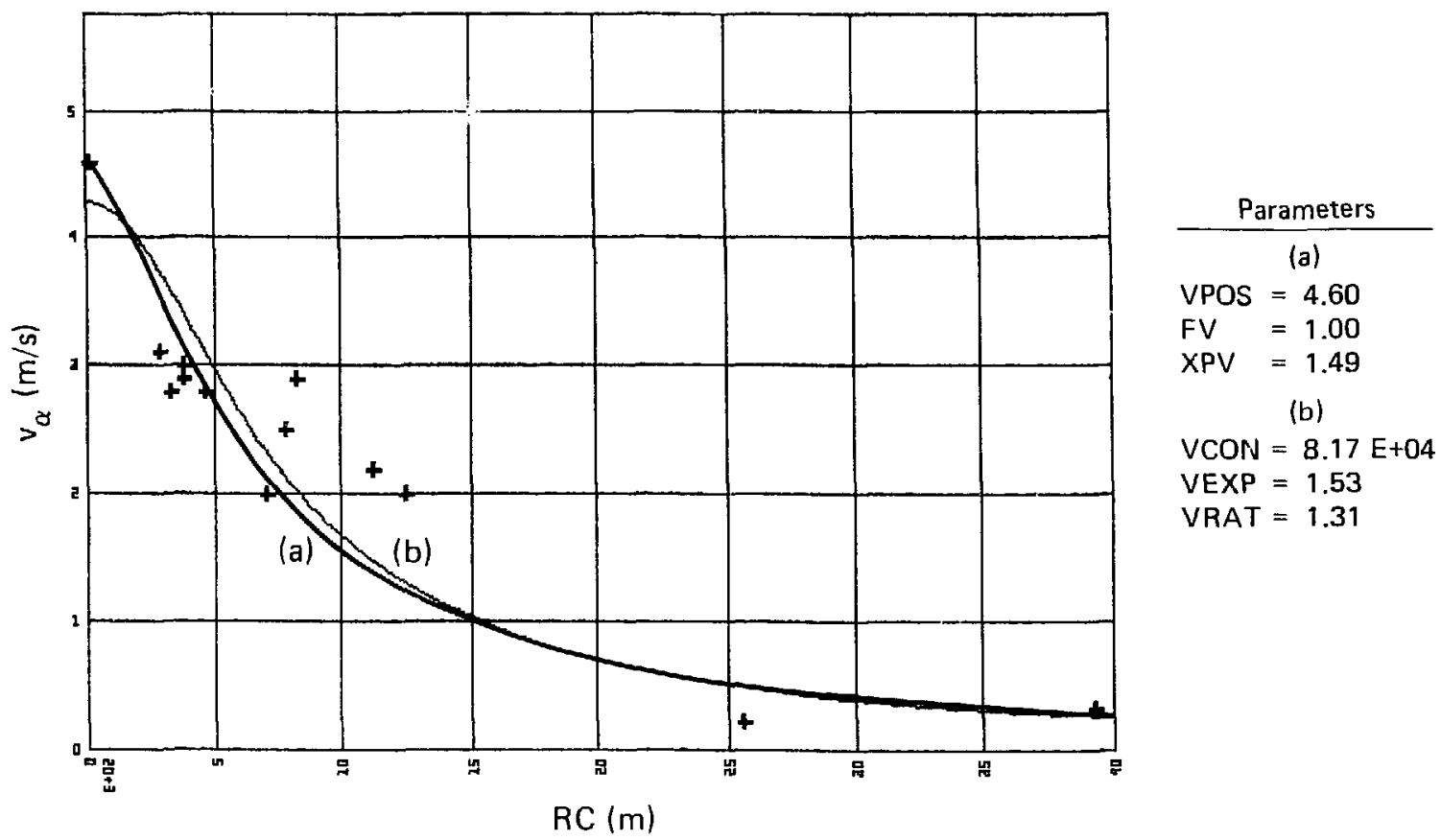

Figure 6. Comparison of the (a) Warshaw and (b) Bernreuter radial model fits to the minimal velocity peak $v_{\alpha}$ for Event $A$. $Z B=640.0$ and $Y=1.0$. The sum of squared residuals for curve (a) is 3.3561 and for curve (b) 3.8858 . 


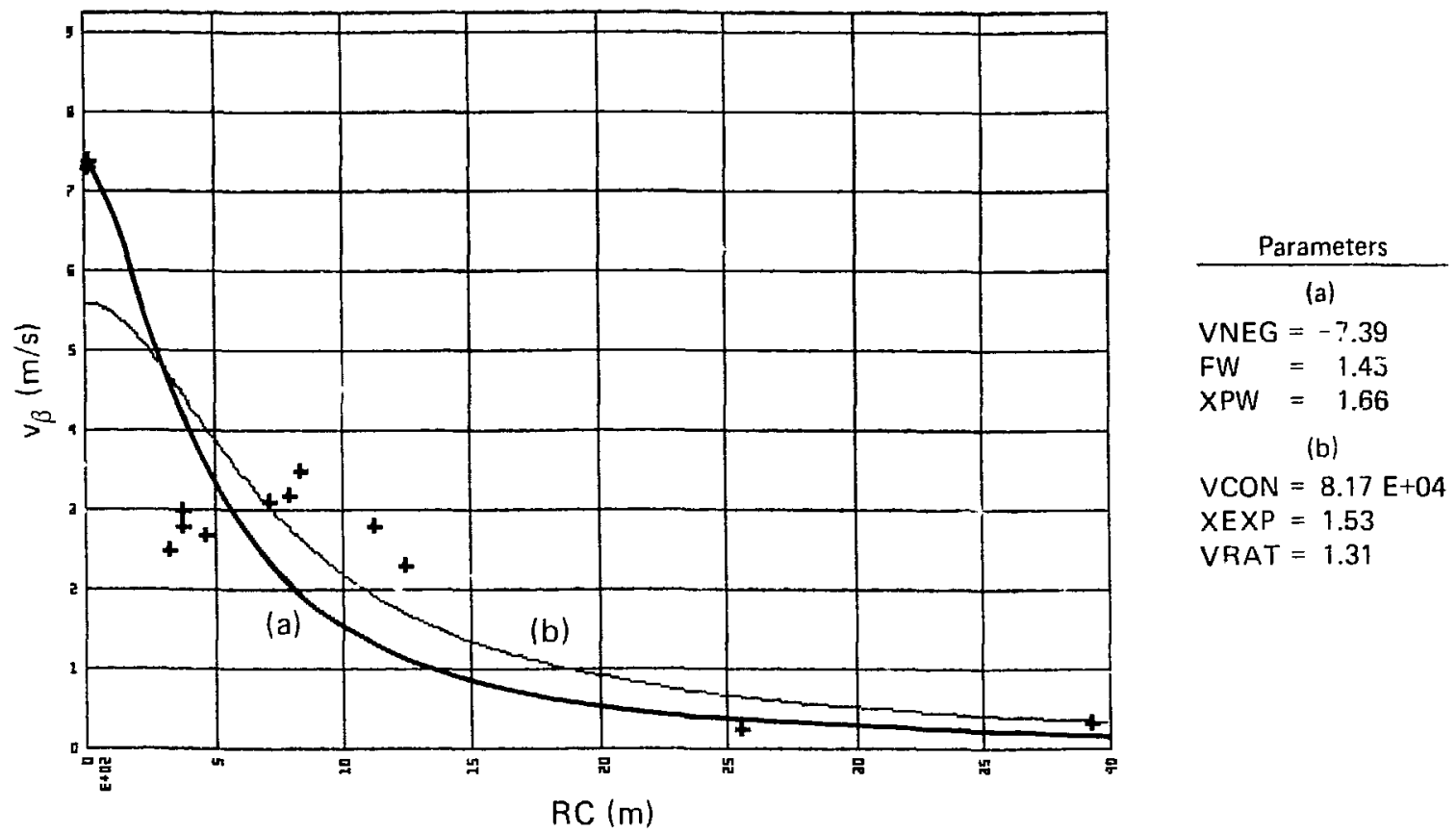

Figure 7. Comparison of the (a) Warshaw and (b) Bern reuter radial model fits to the first negative velocity peak $v_{B}$ for Event $A$. $Z B=640.0$ and $Y=1.0$. The sum of syuared residuals for curve (a) is 14.992 and for curve (b) 19.710 .

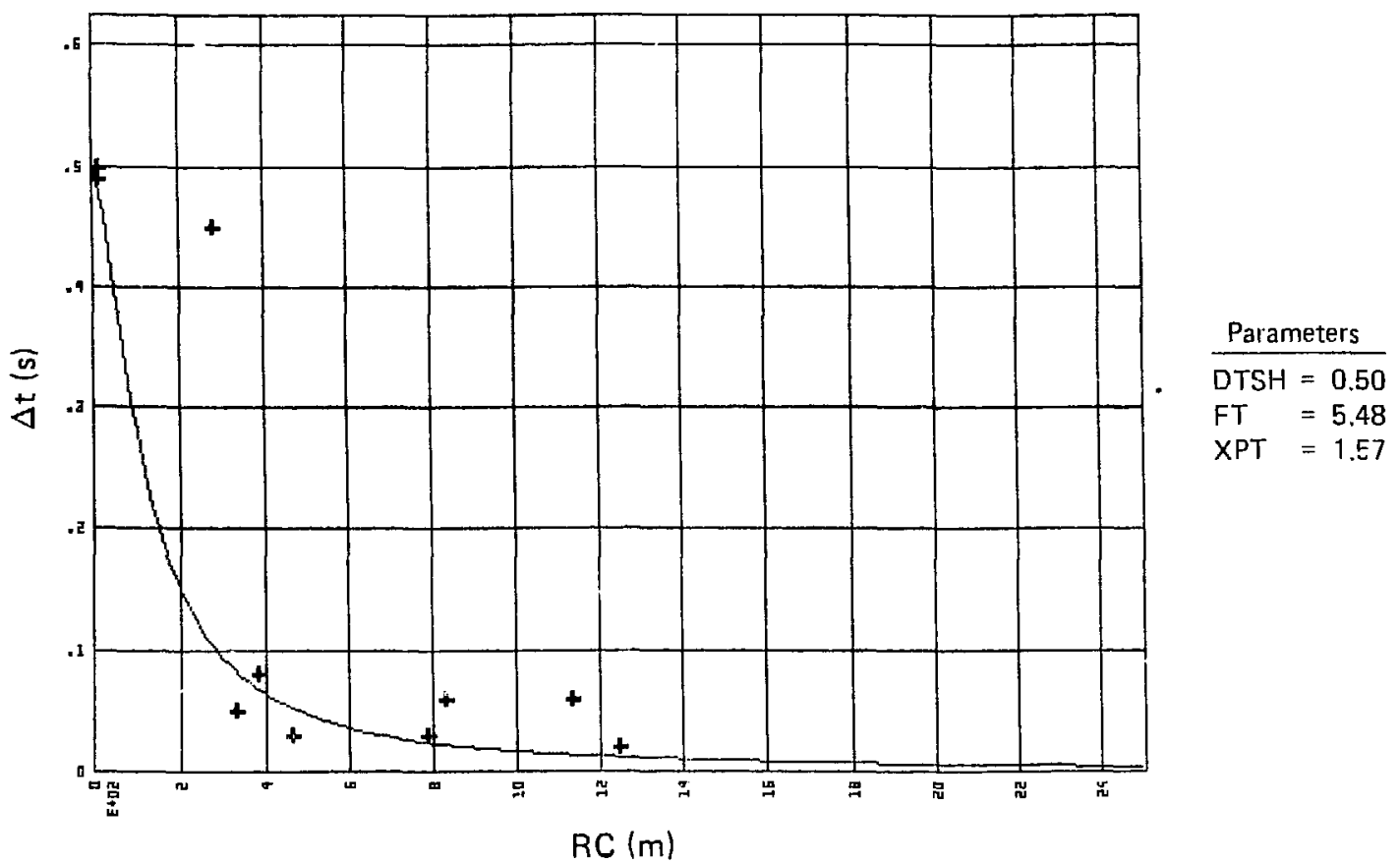

Figure 8. The Warshaw radial fit to the shoulder drop-off time $\Delta t=t_{5}-t_{4}$ for Event $A$. The Bernreuter model is not used for this variable. $\mathrm{ZB}=640.0$ and the sum of squared rosiduals is 0.12850 . 
others it is clipped at $23 \mathrm{~g}$. The correct value is presumably some unknown amount $>23 \mathrm{~g}$. How ever, there is an apparently non-clipped data record at SGZ with a value of $24 \mathrm{~g}$. We can condude that the slapdown peak at $1131 \mathrm{~m}$ is at least as large as at SGZ and fossibly larger. ln addition, there are several data records with smaller radii, smaller magnitudes, and no indication of clipfing $(283 \mathrm{~m}, 329 \mathrm{~m})$. This is contrary to our expectation that acceleration magnitudes generally decrease with distan re from SGZ.

Since the ground motion as a whole has a finite extent, a- necessarily has a radial dependence. However, the clipping of the data prevents us from determining if the for ' 1 of our adial function is appropriate, and if not, what form would be.

To obtain Warshaw parameters for a data set of this type, we must choose some data vilues. It in tempting to replace the clipped data with larger values, but there is no justifi. ble way to determine them. Since we believe that variability is generally small at SCZ. the fit should be cluse to these values. There is already some unknown degree of error if we fit the clipped data, and this error will increase if we try to get closer to the intermediate range data $"$ ith the smaller values. This fit was therefore achiacd by fixing ANEG at 235.2, which is the measured value of the data at SGZ, and by adjusting the other two parameters for a least squares fit to the data as they are, subject to the constraint that the value be small at RCMAX. Berause the chuices of RCMAX and the acederation value ai RCMAX are somewhat arbitrary. these parameters are not unique.

The Bernreuter fit in this case (Fig. 5) is Laken to be the same shape as the Bornreuter fit for a, but multiplied by the factor 3.75 representing the ratio between the data values for a- and a. We do not consider this fit acceptable because it totally ignores the platedu aspect of the data and is probably too large at very large radii such as $3000 \mathrm{~m}$. The excellent match at SGZ is accidental. There are no data points shuwn at the radii of $2560 \mathrm{~m}$ and $3929 \mathrm{~m}$ because no spall is evident there.

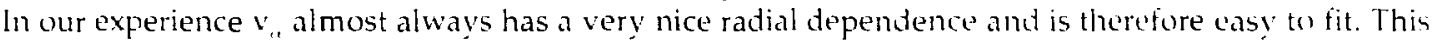
data set is no exception. The Warshaw and Bernreuter fits for ${ }^{\circ}$ (Fig. 6) are similar. The Warshaw model has a slightly better fit at SGZ and the extreme radii. The Bernreuter model fits slightly herter in the intermediate range (283 $\mathrm{m}$ to $1250 \mathrm{~m}$ ).

For this data set RCMAX can be determined in conjunction with fitting $r$. Since the acideration records at $2560 \mathrm{~m}$ and $3929 \mathrm{~m}$ do not show spall and the record at $1250 \mathrm{~m}$ does, we assume that the limit of the extent of spall is between these radii. To estimate its location we took a linear interpolation between the salues of $r$, at $1250 \mathrm{~m}$ and $25 \mathrm{f}^{7}$. to find the radius where the peak velocity is approximately $0.6 \mathrm{~m} / \mathrm{s}$. The result, $2300 \mathrm{~m}$, ad then used as a constraint in the fitting cote: only those Wartiaw parameter sets which satisty $<0.6 \mathrm{~m} / \mathrm{s}$ at $R C=2300 \mathrm{~m}$ are considered acceplable. For future work, we suggest that a more appropriate interpolation would be log-log-linear in the slant distance,${ }^{\prime} R C^{2}+Z^{2}+$ which in this case would have given an estimate of $1890 \mathrm{~m}$.

in practice, there are many local minima with approximately the same sum of squared errors when the no.r-linear least squares routine applies a constraint of this sort. It is thus possible to choose certain desirable features for the fit, such as an exact fit at SGZ. Such a choice is appropriate because the data have more variability in the middle ranges than at SGZ. In other words, we chose to have a better fit at $S G Z$ at the expense of a poorer fit in the intermediate ranges.

The data for $v_{17}$ (Fig. 7) shows a large decrease in the first $200 \mathrm{~m}$. The data between $200 \mathrm{~m}$ and $1250 \mathrm{~m}$ show very little drop-off, so the fit in this region tends to overestimate this data inside $500 \mathrm{~m}$ and underestimate the data bey'ond $500 \mathrm{~m}$. When there is a sharp fall-off in the data close to SGZ, the least squares fit tends to be very stee? as well. This in turn tends to produce overestimates at SGZ. For this reason such data usually must be fit by fixing VNEG at a value which produces a good fit at SGZ, and then making sure that the fit is good at large radii and has a relatively small sum of squared errors.

The Bernreuter fit to $v_{i 1}$ (Fig. 7) would be acceptable if the error at SGZ were not so great. However. since this fit is actually the fit to $v_{0}$ multiplied by a scale factor representing the ratio $v_{.} / v_{*,}$, there is no room to adjust the fit. Another way of looking at this is that the correlation between $v_{*}$ and $v_{3}$ is quite good except at SGZ.

The time interval $\mathrm{st}$ (Fig. 8) is generally very difficult to obtain from the data records. $\Delta t$ turns out to be most useful for determining the width of the velocity peak, so we often obtain values from the velocity records rather than the acceleration records. When the data are obtained from the velocity records there is no precise way to specify where $t_{4}$ and $t_{7}$ occur on the record. We usually take them to be quite close to the 
peak $v_{n}$, which occurs between them. This often produces poor fits to the overall velocity records. In these cases we re-evaluate our initial estimates of $\Delta t$ and adjust the data and parameters at will. This degree of freedom is not appropriate for the other radial parameters.

In virtually every data set we he re seen, the value of $\Delta t$ at SGZ is near $0.4 \mathrm{~s}$, while beyond some critical radius all the values are less than $0.1 \mathrm{~s}$. In some cases the values remain high out to the critical radius and then suddenly drop. This can lead to rather extreme parameter values, especially for the exponent.

The values of the ratio parameters were obtained by making first guesses and then adjusting for good fit. A discussion of the considerations involved in this process is found in Section 4.

\subsection{Velocity Record Fits}

Table 4 provides the complete set of parameters used to calculate these model ground motions. Table 5 lists the azimuth and distance from SGZ for each data record. Figures $9 a-m$ are plots showing the model velocities superimposed on the velocity data records for Event $A$.

Table 4. Parameter values for Event $A$ used to calculate the model fits shown in Figs. 9a-m and $18 \mathrm{c}-\mathrm{d}$.

\begin{tabular}{|c|c|c|c|c|c|c|c|}
\hline \multicolumn{2}{|c|}{$\begin{array}{c}\text { Warshaw } \\
\text { radial }\end{array}$} & \multicolumn{2}{|c|}{ Ratio } & \multicolumn{2}{|c|}{$\begin{array}{l}\text { Bernreuter } \\
\text { radial }\end{array}$} & \multicolumn{2}{|c|}{ Geological } \\
\hline APOS & 47.40 & F1 & 0.85 & $\mathrm{ACON}$ & $2.61 E+05$ & ZB & 640.0 \\
\hline FA & 0.32 & F2 & 0.75 & AEXP & 1.29 & VB & 1735.0 \\
\hline XPA & 9.24 & F3 & 0.65 & ARAT & 3.75 & RCMAX & 2300.0 \\
\hline ANEG & 235.20 & F4 & 0.41 & VCON & $8.17 E+04$ & & \\
\hline FB & 0.42 & F5 & 0.67 & VEXP & 1.53 & & \\
\hline XPB & 6.16 & F6 & 0.16 & VRAT & 1.31 & & \\
\hline VPOS & 4.60 & F7 & 0.85 & $\mathbf{Y}$ & 1.0 & & \\
\hline FV & 1.00 & F8 & 0.66 & & & & \\
\hline XPV & 1.49 & & & & & & \\
\hline VNEG & -7.39 & & & & & & \\
\hline FW & 1.45 & & & & & & \\
\hline XPW & 1.66 & & & & & & \\
\hline DTSH & 0.50 & & & & & & \\
\hline FT & 5.48 & & & & & & \\
\hline XPT & 1.57 & & & & & & \\
\hline
\end{tabular}

Table 5. Accelerometer locations for Event A.

\begin{tabular}{rccc}
\hline $\begin{array}{c}\text { Radius RC } \\
(\mathrm{m})\end{array}$ & Azimuth & $\begin{array}{c}\text { Figure } \\
\text { shown }\end{array}$ & Spall \\
\hline 15 & SW & $9 \mathrm{a}$ & yes \\
15 & SE & $9 \mathrm{~b}$ & yes \\
283 & SW & $9 \mathrm{c}$ & yes \\
329 & $\mathrm{NW}$ & $9 \mathrm{~d}$ & yes \\
385 & $\mathrm{~S}$ & $9 \mathrm{e}$ & yes \\
466 & SE & $9 \mathrm{f}$ & yes \\
707 & SW & $9 \mathrm{~g}$ & yes \\
792 & SE & $9 \mathrm{~h}$ & yes \\
836 & NE & $9 \mathrm{j}$ & yes \\
1131 & SW & $9 j$ & yes \\
1250 & NE & $9 k$ & yes \\
2560 & SE & $9 l$ & no \\
3929 & SE & $9 m$ & no \\
\hline
\end{tabular}


Perhaps the most disconcerting feature of this data is that, in 8 of the 11 data records with observed spall, the velocity toes not return to zero. (See Figs. 9b, c, and e.) This clearly indicates that there may be serious problems in instrument calibration or in data collection, reduction, and presentation. Since these symptoms are present in every data set we have seen, it is equally clear that a full understanding of ground sufface behavior during a UGT will not be possible until better data are available. These problems greatly hinder our use of the model. However, since the data prior to slapdown are usually very good, we
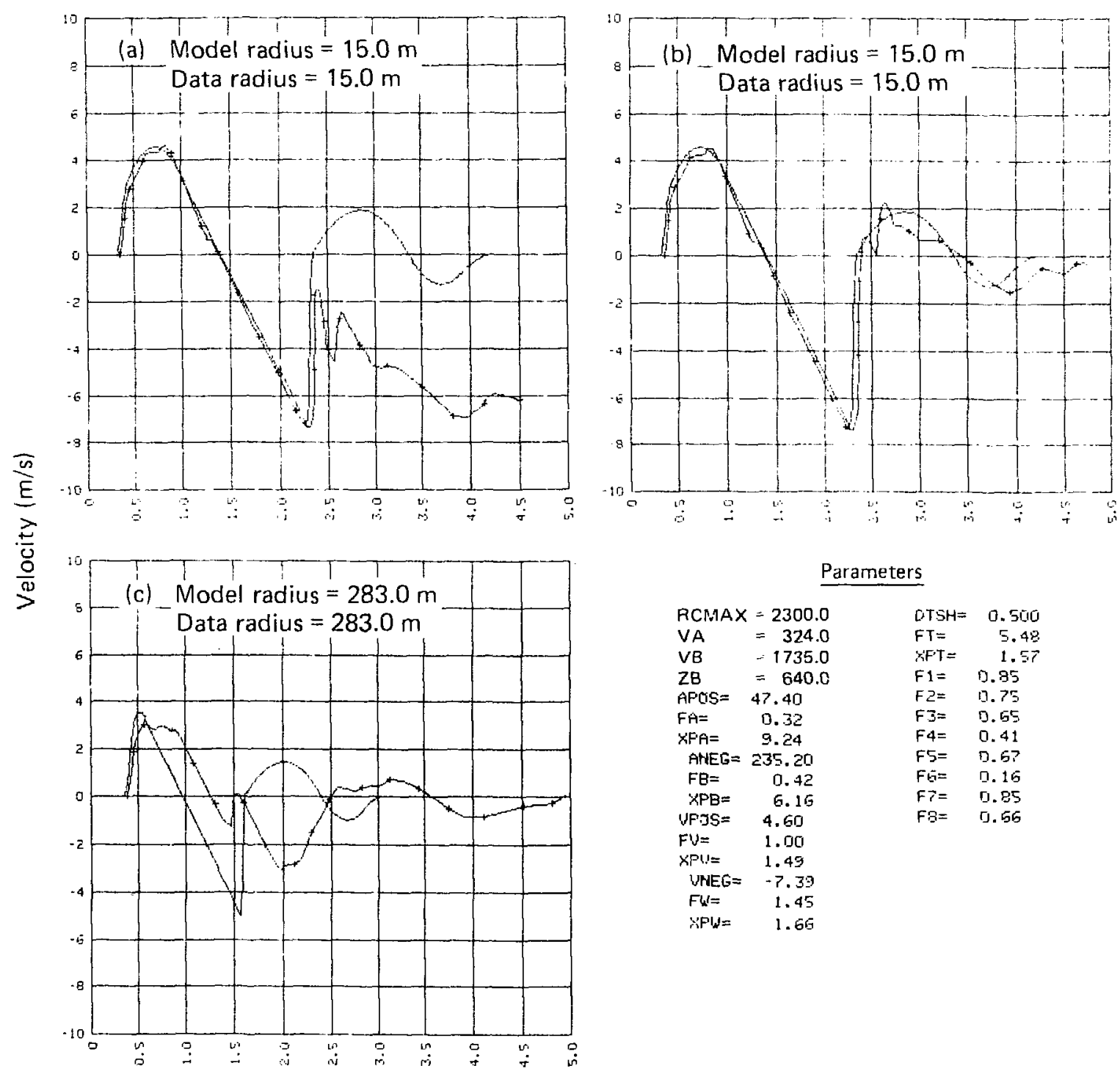

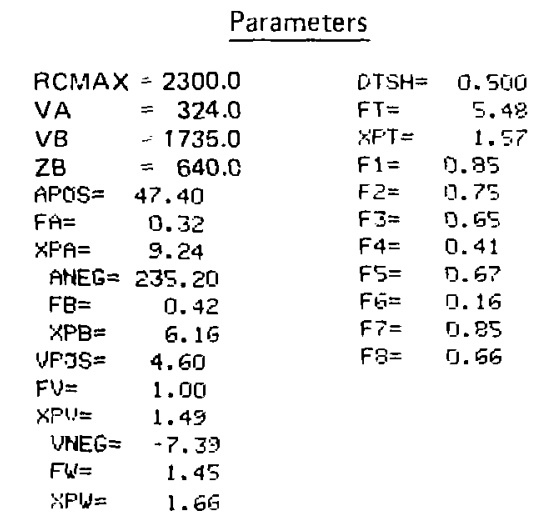

Time (s)

Figure 9. Velocity time history fits for Event $A$ at various distances from SGZ. Each figure (a) through $(\mathrm{m})$ shows the fit of the model velocity record to an accelerometer's data velocity record at a different radius. 
suspect that the extreme forces occurring during slapdown may have been enough to alter the accterometer calibration in these cases.

With regard to both $v_{\alpha}$ and $v_{\beta}$ the choice of radial fit can easily be seen in the superimposed plots. For instance, the fits are quite good at SGZ (Figs. 9a-b), whereas the intermediate ranges show deviations of varying magnitudes. A fit such as the one at $1250 \mathrm{~m}$ (Fig. 9k) may look rather bad when viewed individually, but by referring to the radial fits for $v_{c k}$ and $v_{\beta}$, we can see that these deviations were necessary in order to satisfy other considerations.

In cases where the radial fit is a global minimum, we can indeed claim that any improvement at a particular radius will cause an even larger deterioration of the fit of at least one other radius. In cases where the radial fit is a local minimum due to some constraint, there may be several competing fits. It may

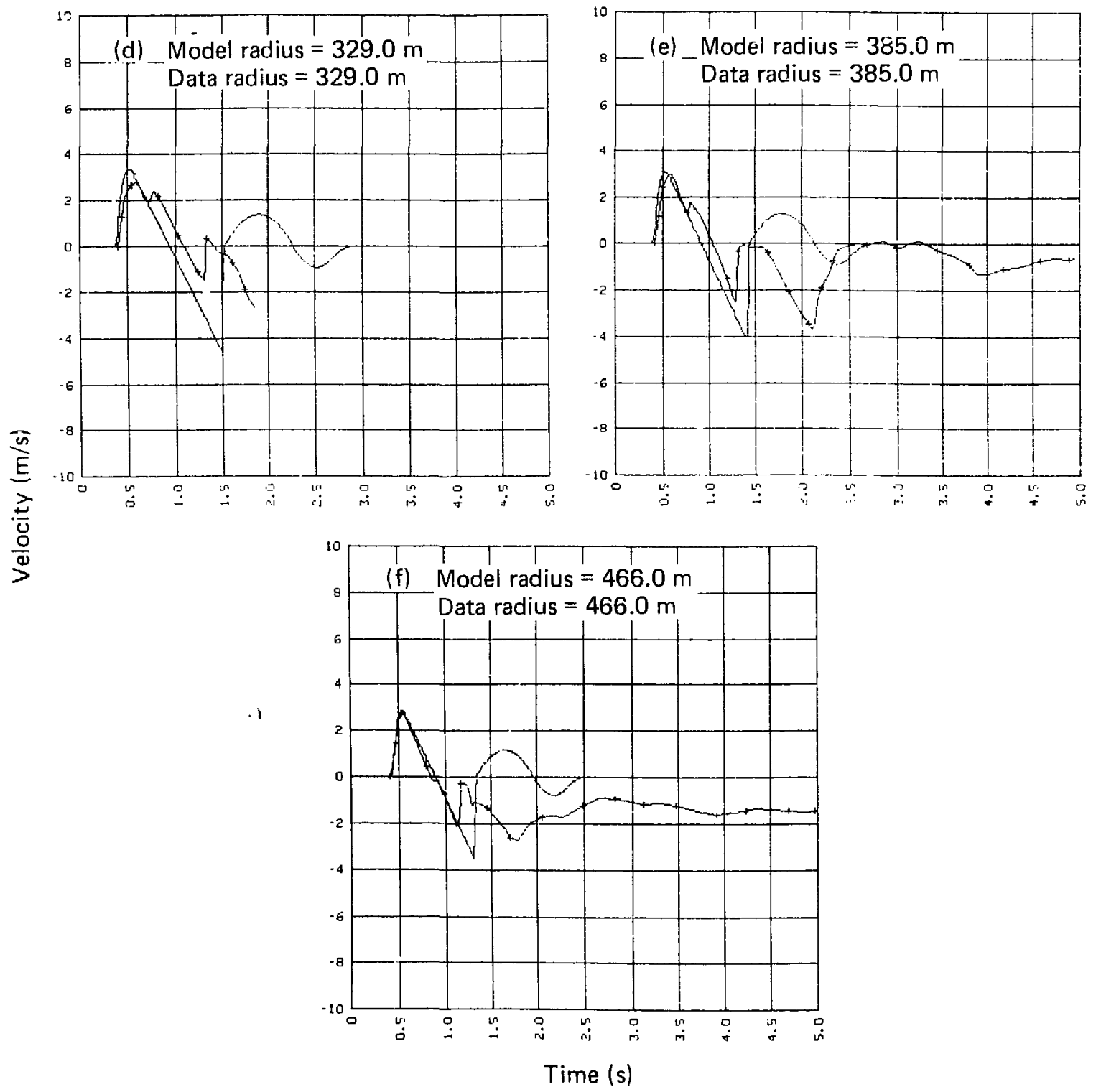

Fig. 9 continued. 
then be possible to improve the fit at a particular location without decreasing the overall sum of squared errors. Whenever this is the case, we choose to give priority to SGZ.

The evaluation of the fit after slapdown, which depends primarily on the ratio parameters, presents problems. It is usually impossible to do at those radii for which the velocity data does not return to zero. If the velocity records that do return to zero have shapes too different from our model, evaluation is difficult or impossible. For this data set only the data record at $15 \mathrm{~m} \mathrm{SE}$ (Fig. 9b) is useable, so we judge the ratio parameters acceptable because the fit to this record is acceptable.
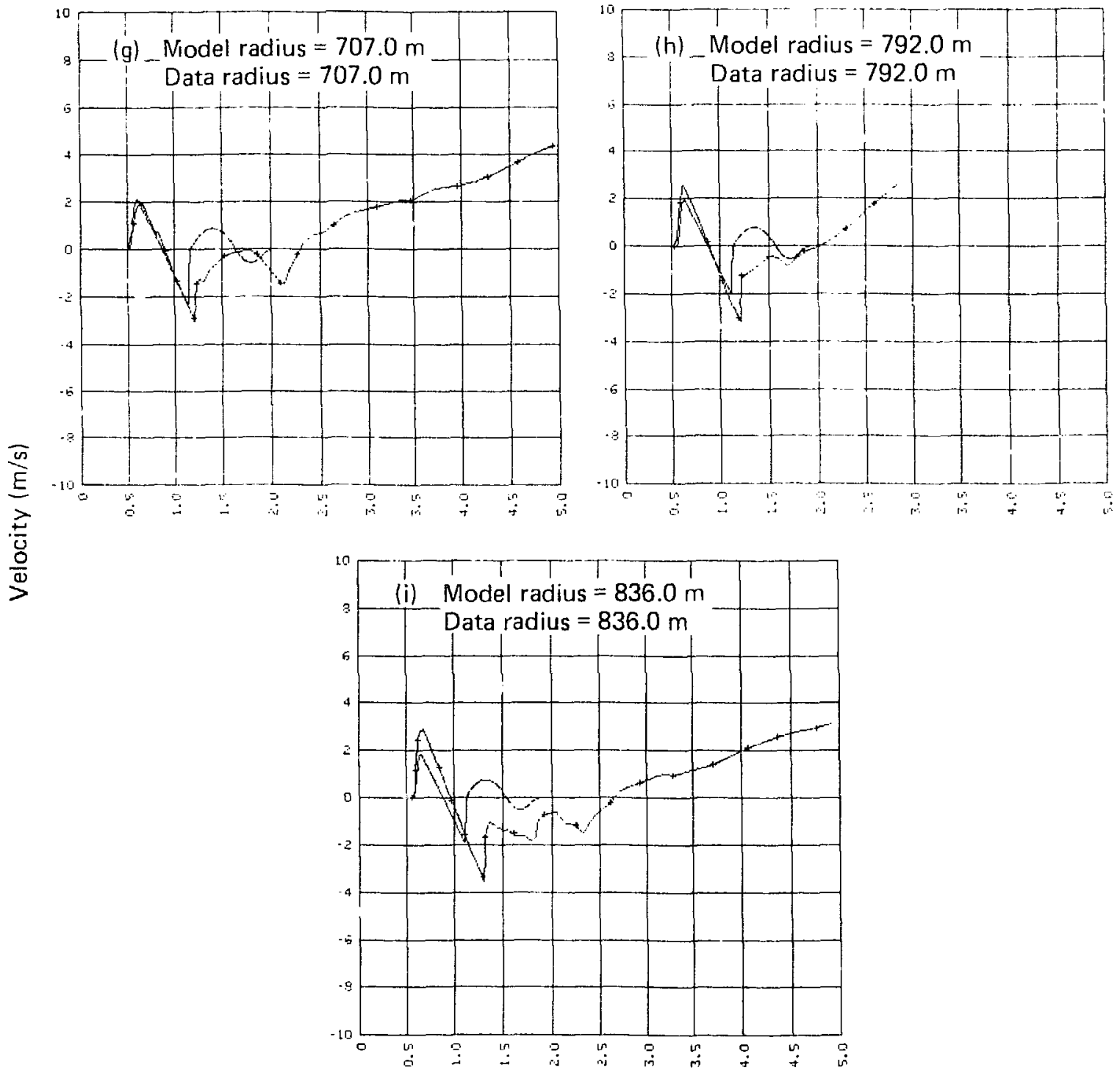

Time (s)

Fig. 9 continued. 

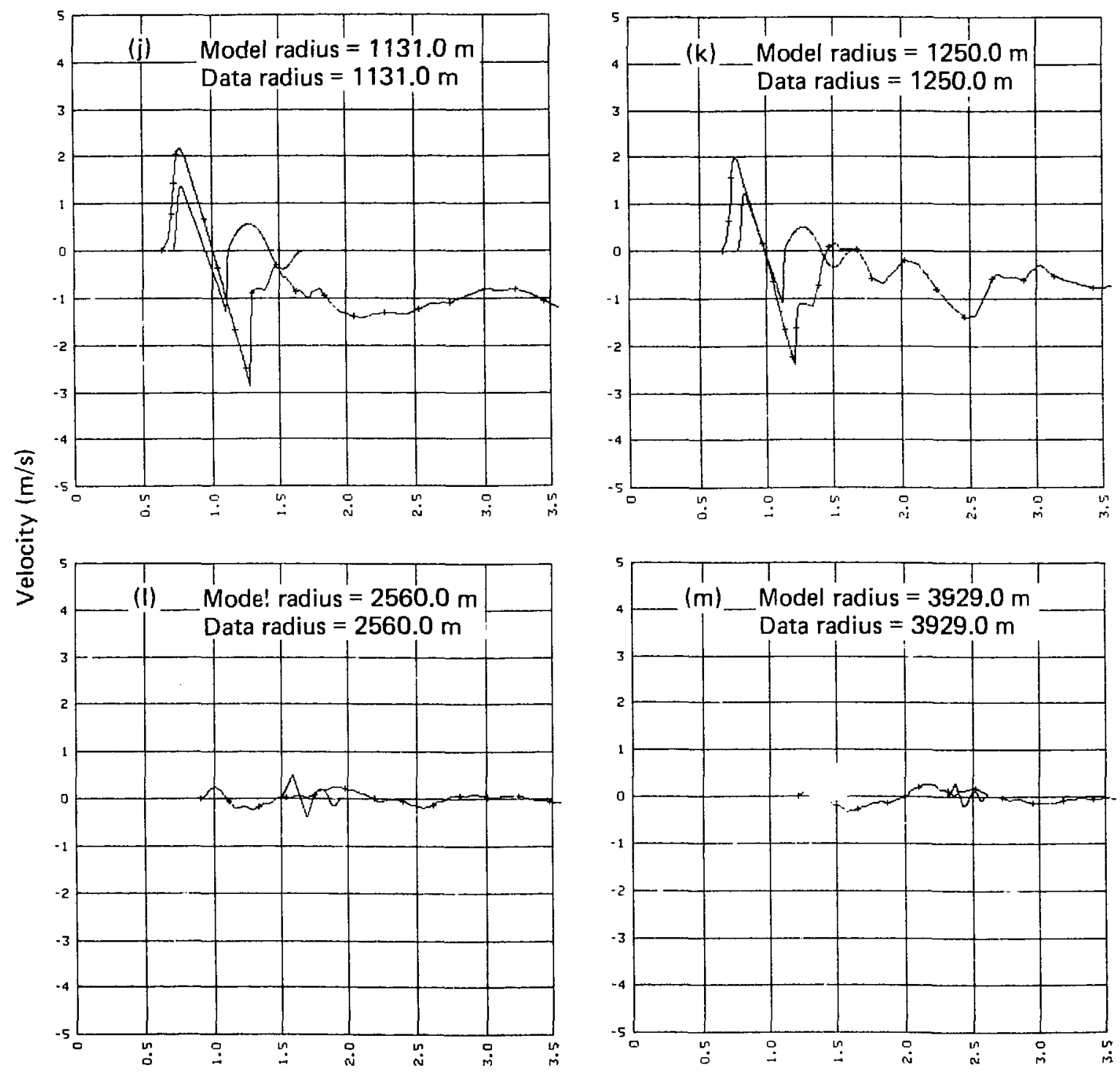

Time (s)

Fig. 9 continued. 


\section{Section 3. Model Fits to Event B}

This section shows how our model fits the data from Event B. Section 3.1 contains the radial parameter fits in Figs. 10-14, and Section 3.2 contains the superimposed velocity plots. The Bernreuter fits were calculated using the value $Y=1.0$.

\subsection{Radial Modei Fits}

Figure 10 show's the Bernreuter fit to the data for $a_{2}$. The unconstrained Warshaw fit, not shown here, is essential!y the same. Clearly, the lack of data beyond $701 \mathrm{~m}$ causes the least squares fit to predict an absurdly large value for $a_{2}$ at large radii. Fits of this sort can be improved by introducing estimates of data with small values at large radii (for the Bernreuter model) or applying a magnitude coustraint (for the Warshaw model), but we must be able to decide where and how small. This is difficult, because all of the data records appear to be well within the spall zone. Thus, we have no direct way of estimating the enirul of spall and no way of estimating RCMAX.

In some of the first data we looked at for other events, the extent of spall appeared to be approximately five times the buria! depth. Although we have since come to believe that using this rule oi thumb generally produces an overestimate, we used it here and obtained RCMAX $=3500 \mathrm{~m}$. For the magnitude constraint at RCMAX we used acceleration and velocity values comparable to those observed in other data sets, such as Event $A$, which had data at comparably large radii. Since this procedure leaves considerable room for many choices of RCMAX and magnitude at RCMAX, these parameters are not unique in any' sense. Other parameter sets could easily produce equally good fits. It is worth noting here that we onl., use the $0.6 \mathrm{~m} / \mathrm{s}$ criterion for determining RCMAX when we have enough ciata to interpolate.

The Warshaw and Bernreuter fits to $a_{2}$ (Fig. 11) were obtained using the constraint and estimated data, respectively. Clearly, the Warshaw fit is better.

The data for a- (Fig. 12) suggest rather strongly that something is wrong, but there are no indications on the data records as 10 whether the problem is with the data at SGZ or at $700 \mathrm{~m}$. Ihe Warshaw fit was obtained only by applying the constraint. Based on our experience with other data sets, we are inclined to be suspicious of the SGZ data; this is why we have settled for a fit that badly overestimates at SGZ. The Bernreuter fit is not significantly different from the Warshaw fit. The data record at $350 \mathrm{~m}$ was not included in the fitting procedure, since it showed double (and perhaps triple) spall. When this happens it is not clear which slapdown peak to use.

For Event $B$, as for Event $A$, the $v_{t r}$ data shows a nice radial dependence (Fig. 13). The Warshaw fit is slightly better, due mainly to its fit at SGZ. The Warshaw fit predicts $0.6 \mathrm{~m} / \mathrm{s}$ at $R C=2000 \mathrm{~m}$, which suggests that the fit is satisfactory even if we overestimated the extent of spall by as much as $1500 \mathrm{~m}$.

Both of the fits to $v_{i f}$ are satisfactory (Fig. 14). Since they are so alike, the choice of which to use is almost arbitrary. We chose the Warshaw fit because it does a better job with the three data records at $701 \mathrm{~m}$. The data record at $350 \mathrm{~m}$ was not used for $v_{d j}$, since it was not clear where $v_{i j}$ occurred on the velocity record.

The data shown on Fig. 15 are our initial guesses for $\Delta t$. They produced very bad fits of the fiist velocity peak widths on the superimposed velocity plots. The fit is to the adjusted data, which is not shown. The adjustment procedure is as follows. Taking each data record separately, we determine by trial and error the value of $\Delta t$ that produces a good fit of the velocity peak width. These new values are then fit as if they had been obtained directly from the data records. This approach was very successful with this data set. 


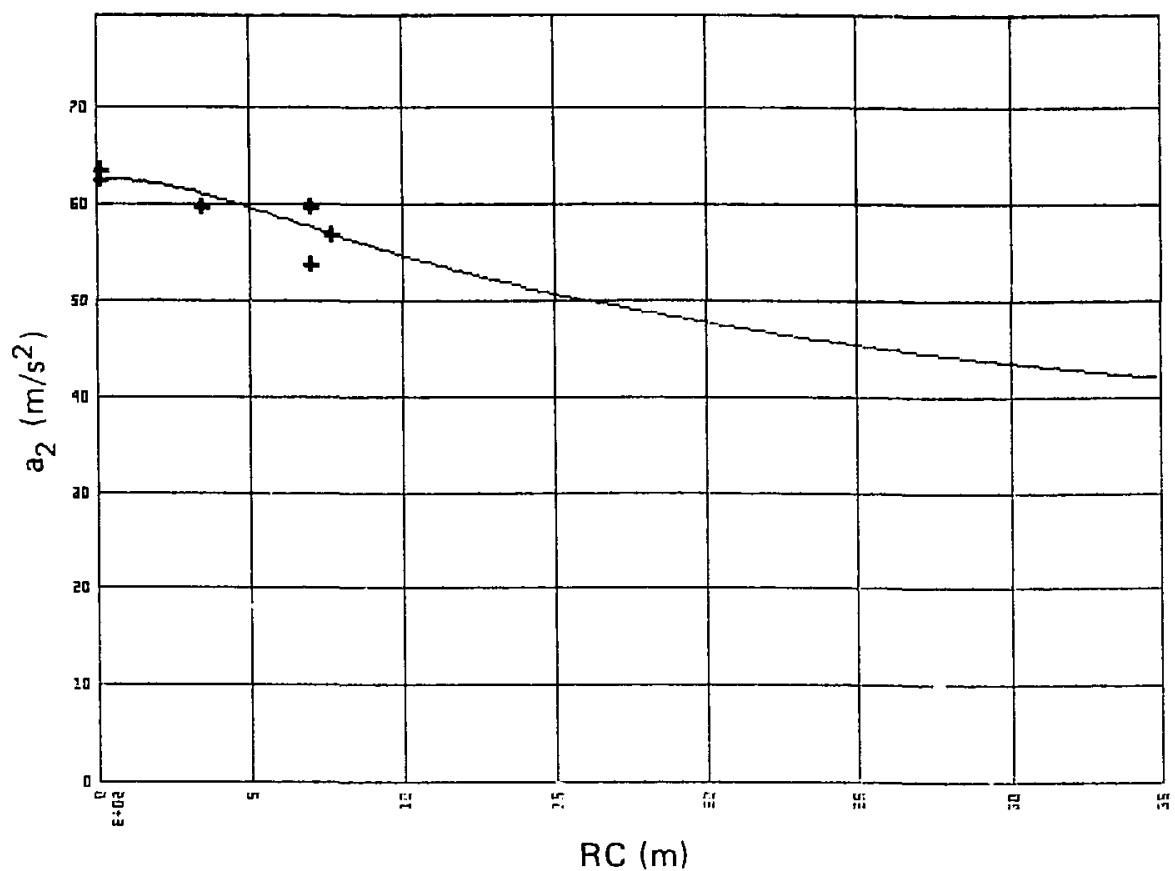

\begin{tabular}{lr} 
Parameters \\
\hline ACON $=318.68$ \\
AEXP $=$ & -0.248 \\
ARAT $=$ & 2.133
\end{tabular}

Figure 10. The Bernreuter unconstrained radial fit to the initial acceleration peak $a_{2}$ for Event $B$. The data set contained no points at large radii. Without an appropriate constraint, the fit predicts excessively large values of $a_{2}$ at large distances from SGZ. ZB $=701.0$ and $:=1.0$. The sum of squared residuals is 25.717 .

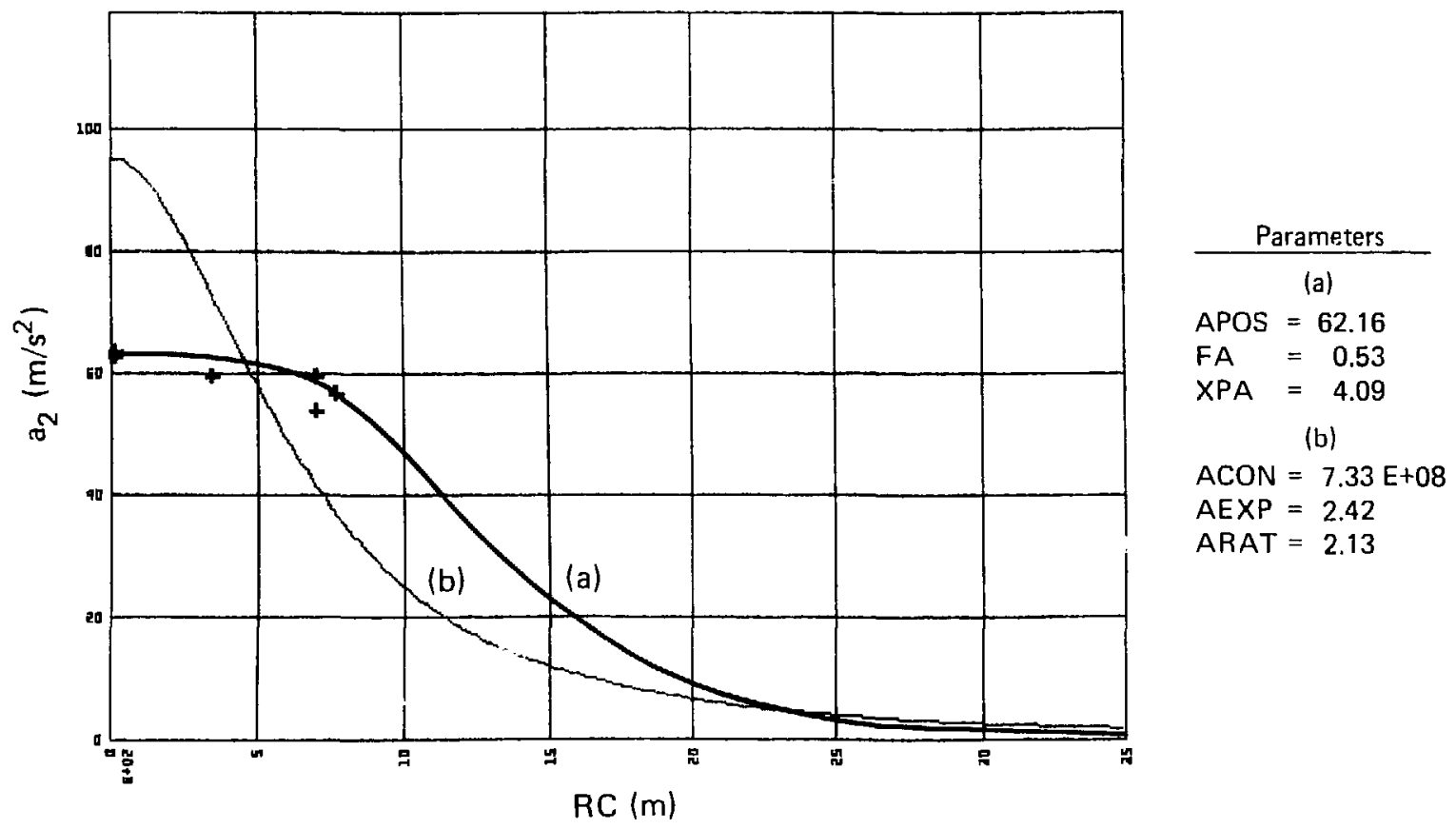

Figure 11. Comparison of the constrained (a) Warshaw and (b) Bernreuter radial model fits to the initial acceleration peak $a_{2}$ for Event $B . Z B=701.0$ and $Y=1.0$. The sum of squared residuals for curve (a) is 1388.0 , and for curve (b) 3470.0 . 


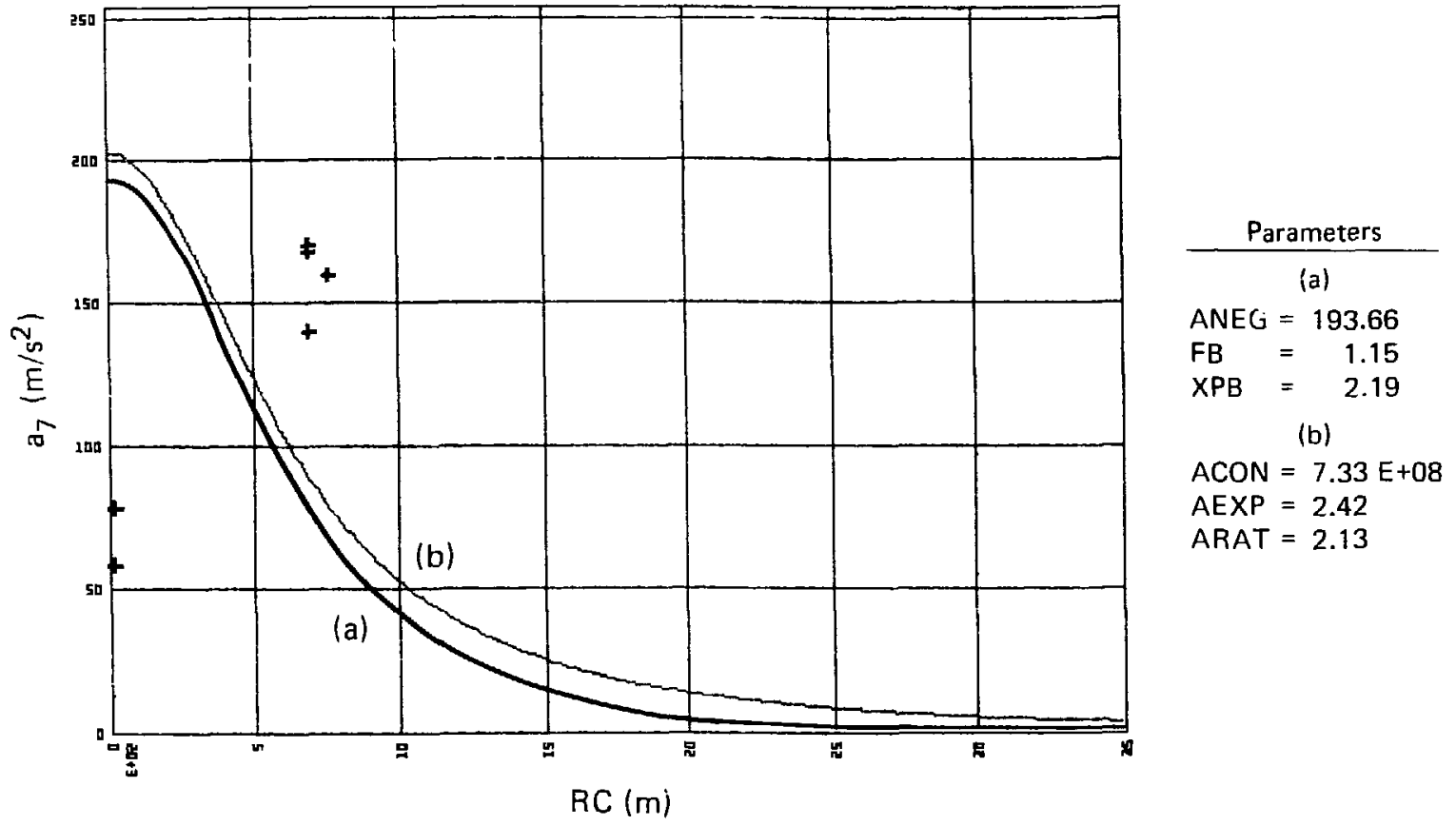

Figure 12. Comparison of the constrained (a) Warshav' and (b) Bernreuter radial model fits to the initial acceleration peak $a_{7}$ for Event $B . Z B=701.0$ and $\underline{v}=1.0$. The sum of squared residuals for curve (a) is 52830.0, and for curve (b) 58914.0.

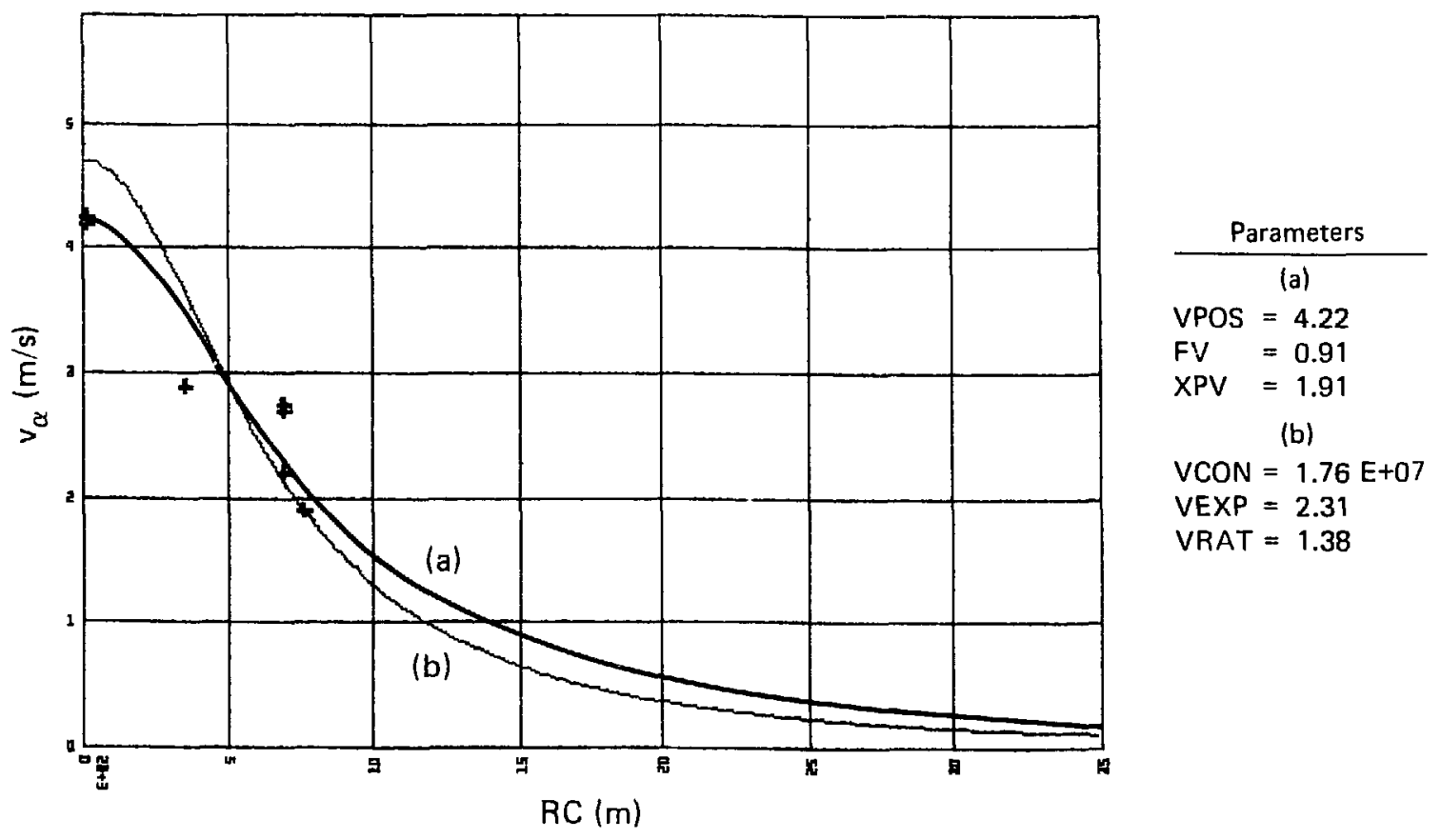

Figure 13. Comparison of the constrained (a) Warshaw and (b) Bernreuter radial model fits to the initial acceleration peak $v_{\alpha}$ for Event $B . Z B=701.0$ and $Y=1.0$. The sum of squared residuals for curve (a) is 0.79862 , and for curve (b) 1.7369 . 


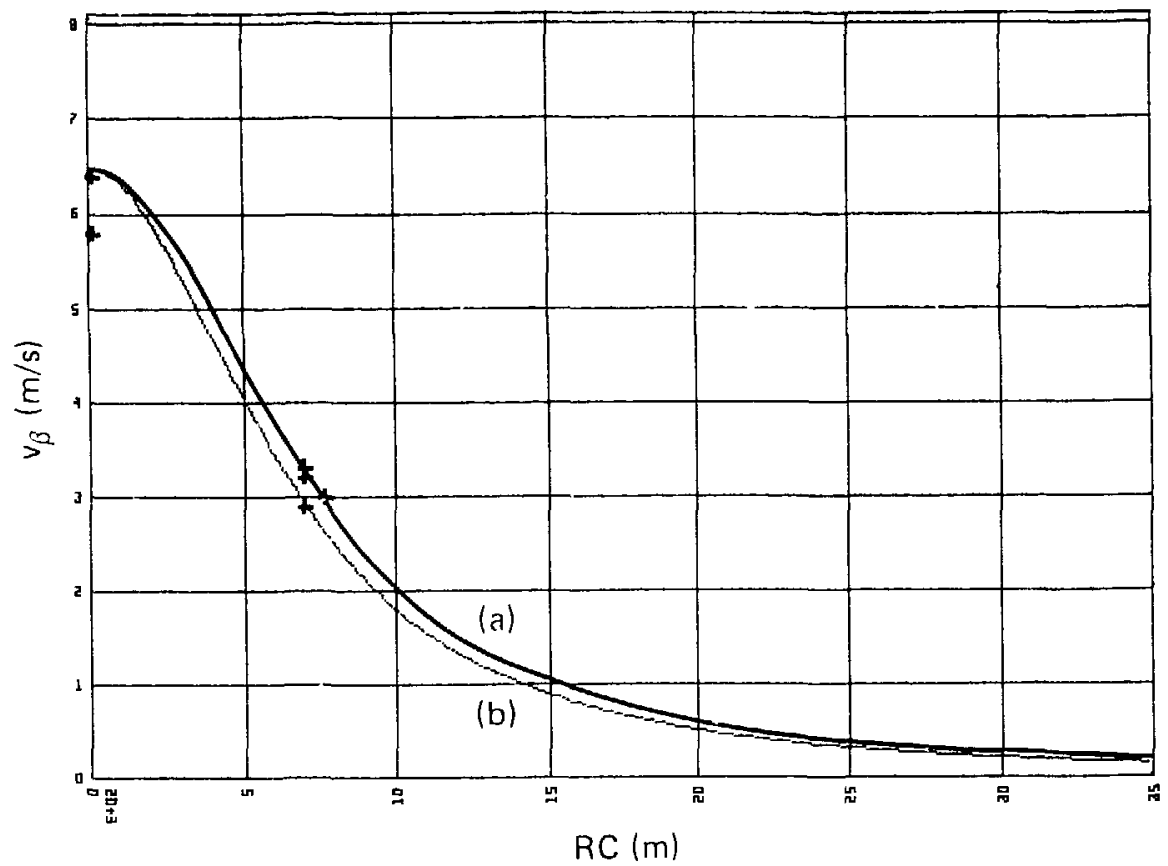

Parameters

(a)

VNEG $=-6.40$

$\mathrm{FW}=1.01$

$X P W=2.18$

(b)

VCON $=1.76 E+07$

VEXP $=2.31$

VRAT $=1.38$

Figure 14. Comparison of the constrained (a) Warshaw and (b) Bernreuter radial model fits to the initial acceleration peak $v_{B}$ for Event $B . Z B=701.0$ and $Y=1.0$. The sum of squared residuals for curve (a) is 0.47155 , and for curve (b) 0.85540 .

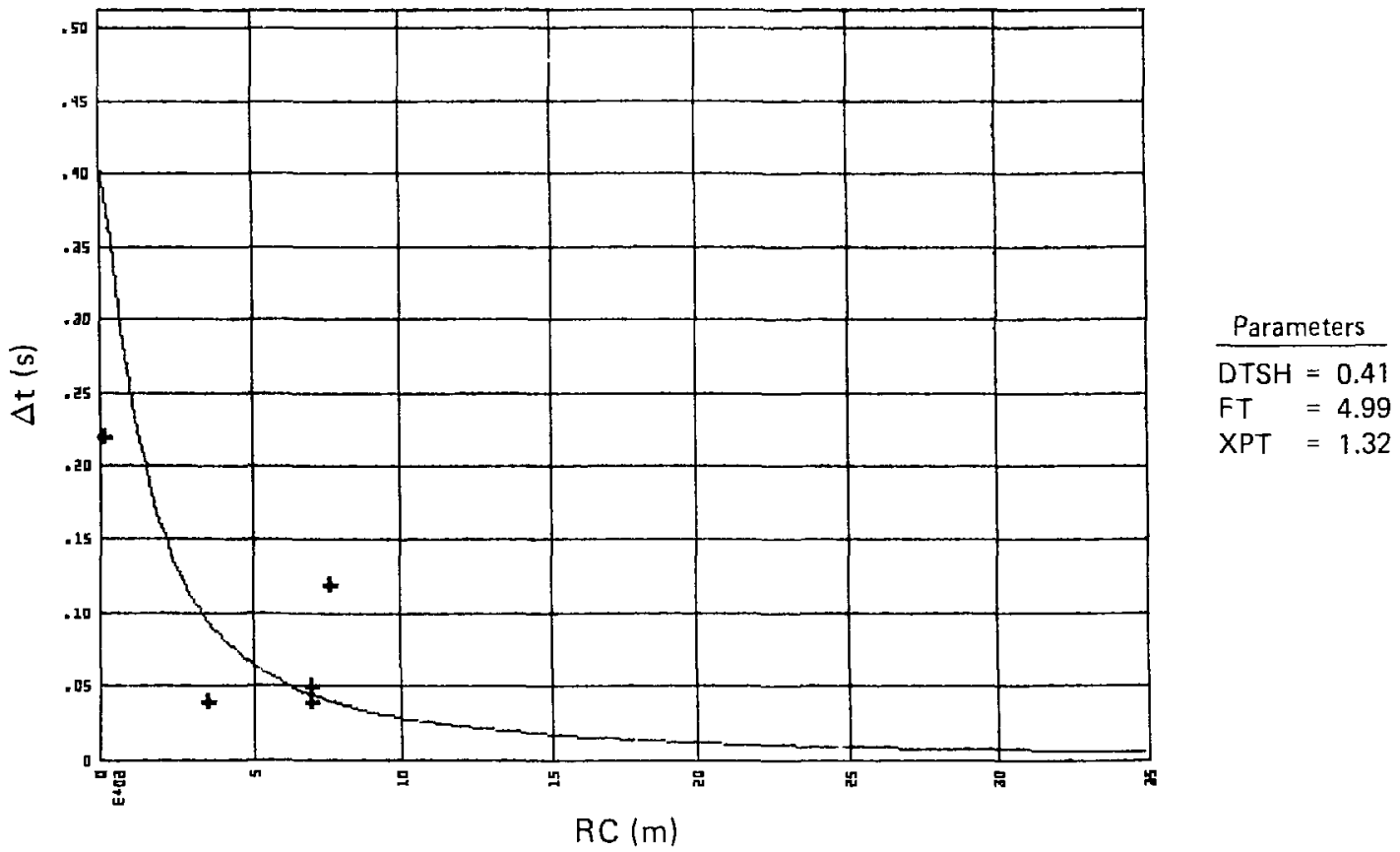

Figure 15. Our initial guesses for $\Delta t$ using the Warshaw radial fit. We used adjusted data, which we produced by taking each data record separately and determining by trial and error the value of $\Delta t$ that produces a good fit of the velocity peak width. $Z B=701.0$ and $Y=1.0$. The sum of squared residuals is $7.735 \mathrm{E}-02$. 


\subsection{Velocity Record Fits}

Table 6 lists the parameters used to calculate the superimposed velocity plots found in Figs. 16a-g. Table 7 is a list of the station radii and azimuths. All are within the spall zone.

We were able to obtain good to excellent fits to the first positive and negative peaks $\left(v_{\alpha}\right.$ and $\left.v_{B}\right)$, both in magnitude and width, at all locations except $350 \mathrm{~m} / \mathrm{s}$, where it is not clear what a good fit would be. The second two velocity peaks $\left(v_{\gamma}\right.$ and $v_{s}$ ) are not quite so good. We should mention that when there are few data records, and several of them at the same radii, fitting all of them is relatively easy.

Table 6. Parameter values for Event $B$ used to calculate the model fits shown in Figs. 16a-g and 20.

\begin{tabular}{|c|c|c|c|c|c|c|c|}
\hline \multicolumn{2}{|c|}{$\begin{array}{c}\text { Warshaw } \\
\text { radial }\end{array}$} & \multicolumn{2}{|c|}{ Ratio } & \multicolumn{2}{|c|}{$\begin{array}{l}\text { Bernreuter } \\
\text { radial }\end{array}$} & \multicolumn{2}{|c|}{ Geological } \\
\hline APOS & 62.16 & F1 & 1.13 & ACON & $7.33 \mathrm{E}+08$ & $\mathbf{Z B}$ & 701.0 \\
\hline FA & 0.53 & F2 & 0.75 & AEXP & 2.42 & VB & 1843.0 \\
\hline $\mathbf{X P A}$ & 4.09 & F3 & 0.58 & ARAT & 2.13 & RCMAX & 3500.0 \\
\hline ANEG & 193.66 & F4 & 0.34 & VCON & $1.76 \mathrm{E}+07$ & & \\
\hline FB & 1.15 & F5 & 0.65 & VEXP & 2.31 & & \\
\hline XPB & 2.19 & F6 & 0.29 & VRAT & 1.39 & & \\
\hline VPOS & 4.22 & F7 & 0.92 & $\mathrm{Y}$ & 1.0 & & \\
\hline FV & 0.91 & F8 & 0.44 & & & & \\
\hline XPV & 1.91 & & & & & & \\
\hline VNEG & -6.40 & & & & & & \\
\hline FW & 1.01 & & & & & & \\
\hline XP'N & 2.18 & & & & & & \\
\hline DTSH & 0.41 & & & & & & \\
\hline FT & 4.99 & & & & & & \\
\hline $\mathbf{X P T}$ & 1.32 & & & & & & \\
\hline
\end{tabular}

Table 7. Accelerometer locations for Event B.

\begin{tabular}{rlc}
\hline $\begin{array}{c}\text { Radius RC } \\
(\mathrm{m})\end{array}$ & Azimuth & $\begin{array}{c}\text { Figure } \\
\text { shown }\end{array}$ \\
\hline 15 & S & $16 \mathrm{a}$ \\
15 & SW & $16 \mathrm{~b}$ \\
350 & S & $16 \mathrm{c}$ \\
701 & NW & $16 \mathrm{~d}$ \\
701 & NE & $16 \mathrm{e}$ \\
701 & SW & $16 \mathrm{f}$ \\
769 & S & $16 \mathrm{~g}$ \\
\hline
\end{tabular}



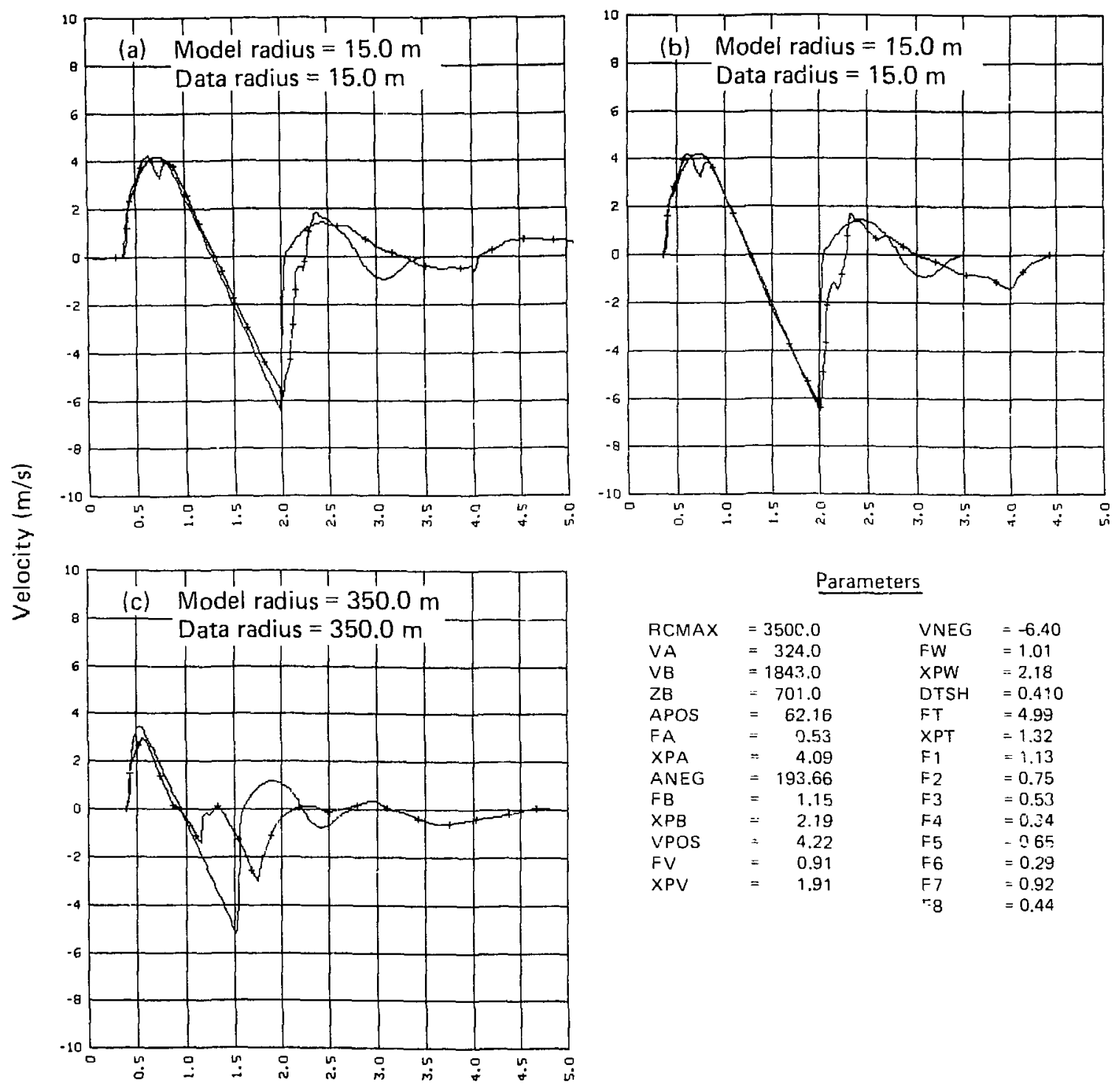

Parameters

\begin{tabular}{|c|c|c|c|}
\hline RCMAX & $=3500.0$ & VNEG & $=-6.40$ \\
\hline VA & $=324.0$ & $F W$ & $=1.01$ \\
\hline VB & $=1843.0$ & XPW & $=2.18$ \\
\hline ZB & $=701.0$ & DTSH & $=0.410$ \\
\hline APOS & 62.16 & FT & $=4.99$ \\
\hline FA & 2.53 & XPT & $=1.32$ \\
\hline XPA & 4.09 & $F_{1}$ & $=1.13$ \\
\hline ANEG & 193.66 & $\mathrm{~F} 2$ & $=0.75$ \\
\hline FB & 1.15 & F3 & $=0.53$ \\
\hline$X P B$ & 2.19 & $\mathrm{~F}_{4}$ & $=0.24$ \\
\hline VPOS & 4.22 & F5 & -065 \\
\hline FV & 0.91 & F6 & $=0.29$ \\
\hline \multirow{2}{*}{ XPV } & 1.91 & $F 7$ & $=0.92$ \\
\hline & & $=8$ & $=0.44$ \\
\hline
\end{tabular}

Time (s)

Figure 16. Velocity time history fits for Event $B$ at various distances (in $m$ ) from SGZ. Each figure (a) through (g) shows the fit of the model velocity record to an accelerometer's data velocity record at a different radius. 

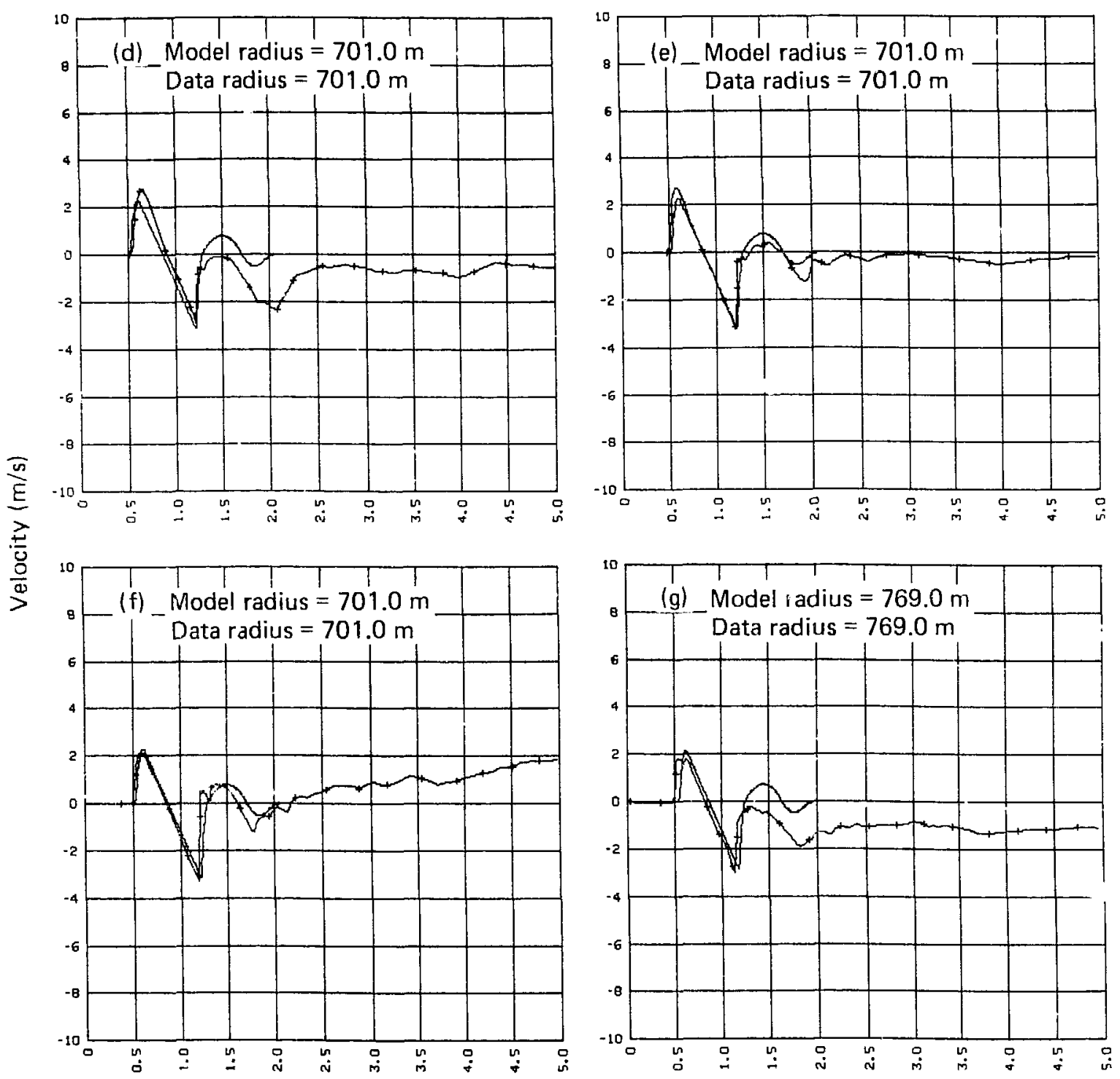

Time (s)

Fig. 16 continued. 


\section{Section 4. Ratio Parameters}

The first ratio parameter, F1, specifies the magnitude of the shoulder following the first acceleration peak. Figure 17 shows schematic examples of the first portion of four typical acceleration records. From these it can be seen that the Warshaw model is evidently too simplistic for these cases.

Figure $17 \mathrm{a}$ is typical of accelerations very close to SGZ. Beyond some radius ( $500 \mathrm{~m}$ for Event $\mathrm{A}$ ) the shoulder has usually disappeared, as seen in Fig. $17 \mathrm{~d}$. The remaining examples are typical of medium ranges. The arrows indicate approximately where the data values for $a_{3}$ and $a_{4}$ should be taken. In the case of Event $A$ only five of the acceleration records yielded values for $a_{3}$. Four of the seven data records for Event $B$ were readily interpretable. The value of $F 1$ is the geometric mean of the useable data. F1 has a small effect on the width of the first velocity peak.

The parameter F2 specifies the magnitudes of the accelerations $a_{b}$ and $a_{y}$. Acceleration records in this region often look more like noise than any easily described function, so the model here is definitely an over-simplification. Obtaining values is very difficult, but if there are any data records from which a value for $a_{s}$ or $a_{9}$ can be plausibly determined, we use their feometric mean for an initial guess for F2. The main function of F2 is to determine, along with F4, the time interval $t_{9}-t_{8}$.

$\mathrm{F} 4$ determines the valie of $v_{i}$, the second velocity peak, as a proportion of $v_{11}$. Thus $v_{7}$ has a radial dependence. The geometric mean of the ratio $v_{1} / v_{11}$ is used for F4. Generally, the magnitude of the ground motion will decrease with time, so we expect $\mathrm{F} 4$ to be less than 1.0.

The time interval $t_{9}-t_{8}$ is calculated with the equation

$t_{4}-t_{8}=4 \frac{F 4}{F 2} \frac{v_{11}}{g}$

There are usually at least one or two records with good velocity data after slapdown, so F2 and F4 are adjusted as necessary to obtain a gocd fit for $t_{y}-t_{b}$ on these records. Only one station for Event $A$ qualified (15 m SE, Fig. 9b), so the adjustment procedure was easy. Adjustment was also necessary for Event B.

The parameters F3 and F5 relate in much the same way as F2 and F4. F3 should be small because it represents an acceleration with no radial dependence (in fact, our model requires $\mathrm{F} 3<1.0$ for computational purposes), and F5 should he less than 1.0 for the same reason as is F4. Fits for F3, the final acceleration peak, are usually hard to obtain because the model is excessively simple. The initial guess for F5 will be the geometric mear of the ratios $v_{j} / v_{-}$from those data records which are useable. I3 3 and F5 together control $t_{11}-t_{4}$, the width of the final velocity peak, relative to $t_{9}-t_{s,}$ via the following relationship:

$t_{11}-t_{9}=\frac{F 5(1+F 3)}{2 F 3}\left(t_{9}-t_{8}\right)$

Thus, in practice it is possible to specify the magnitude of the final velocity peak using F5 and then adjust its width with F3 until it matches as well as possible whatever velocity records are clean enough to use.

For Event $B$ six of the seven velocity records were of sufficient quality so that the fit for the final two velocity peaks could be adequately assessed and therefore improved.

The parameters F2, F3, F4, and F5 are collectively adjusted so that good data records are matched by the model, or so that the velocity model looks reasonable if there is no good data.

The parameter F6 specifies $t_{7}-t_{6}$, the slapdown peak rise time, relative to $t_{2}-t_{1}$, the initial peak rise time. The time scale on most of our data records is such that $t_{7}-t_{6}$ cannot be measured with much accuracy. However, it tends to be in the 0.01 to $0.03 \mathrm{~s}$ range. Typical values for $t_{2}-t_{1}$ are 0.05 to $0.09 \mathrm{~s}$. We have found values of $\mathrm{F} 6$ ranging from 0.1 to 0.5 . The observable effects of this parameter on the model velocity are small and limited to the width of the first negative velocity peak.

The parameter F7 specifies the slope of the velocitv between times $t_{7}$ and $t_{6}$. Under conditions of single spall the slope will be $-g$ because the spalled layer is in free-fall, and so F7 is 1.0 . However, it is 


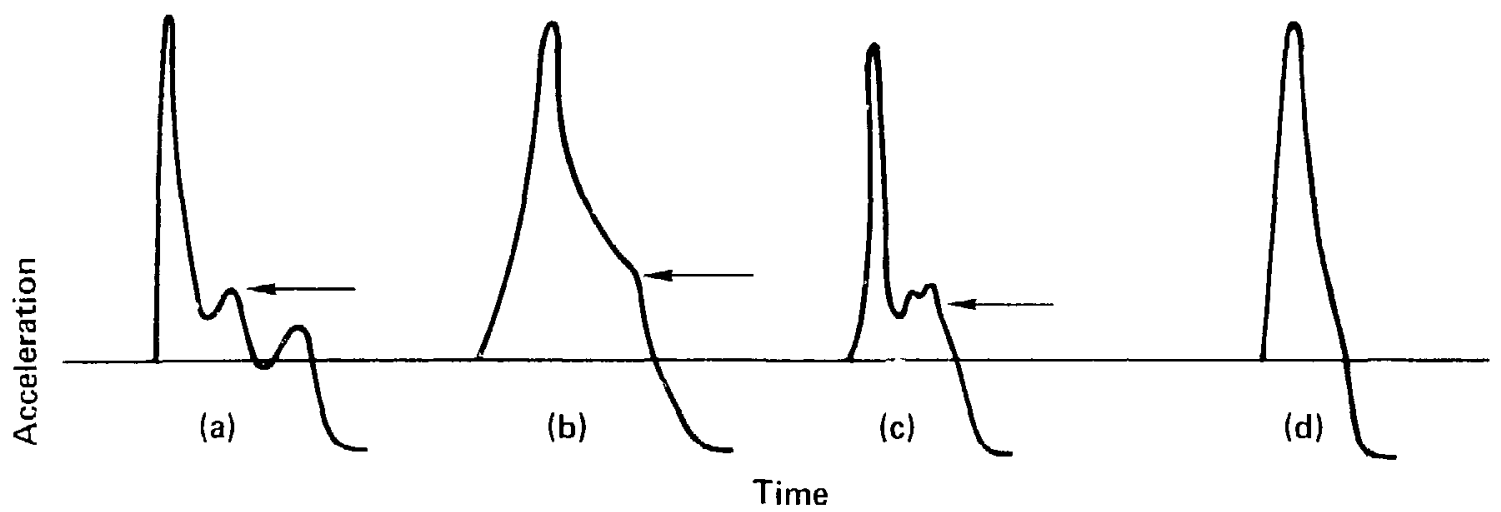

Figure 17. Typical first acceleration peak profiles showing the possible range of shapes. Arrows indicate plausible values for $a_{3}$ or $a_{4}$.

fairly common for there to be some sort of disturbance during this interval (often a double spall) that changes the average acceleration. We adjust F7 to deal with this. Many of the Event $A$ accelerometers recorded an effect of this sort. It is quite pronounced at stations $329 \mathrm{~m}$ and $385 \mathrm{~m}$ (Figs. $9 \mathrm{~d}-\mathrm{e}$ ) as a bump between $v_{n}$ and $v_{b}$ and rather subtle at the two $15 \mathrm{~m}$ stations (Figs. 9a-b). F7 was adjusted to compensate at the $15 \mathrm{~m}$ stations for this disturbance. The fit for Event B was improved by a small adjustment in F7, although in only ont case ( $350 \mathrm{~m}$, Fig. 16c) was this type of disturbance large.

We mentioned earlier that the shoulder between $t_{3}$ and $t_{4}$ disappears as the radius increases. This means that the difference between $v_{3}$ and $v_{c}$ diminishes with radius. Since the disappearance of the shoulder also affects the time interval $t_{5}-t_{4}$, the radial dependence of the relationship between $v_{48}$ and $v_{3}$ should also involve the radial dependence of $t_{5}-t_{4}$. Thus, the parameterization of $v_{3}$ is

$$
\mathrm{v}_{3}=\mathrm{v}_{\mathrm{a}}\left\{1-(1-\mathrm{F} 8)\left[1+\left(\mathrm{FT} \frac{\mathrm{RC}}{\mathrm{ZB}}\right)^{\mathrm{x} \mathrm{TT}}\right]^{-1}\right\}
$$

In essence, F8 specifies the ratio $v_{3} / v_{\alpha}$ at SGZ, and FT and XPT specify the rate at which the ratio $v_{3} / v_{n}$ approaches 1.0 as the radius increases. Since the parameters FT and XPT are already determined by the $t_{5}-t_{4}$ fit, F8 must be determined from the data at SGZ. Strictly speaking, the stations at SGZ do not have $R C=0$, but we consider them close enough. $F 8$ is then the geometric mean of the ratios $v_{3} / v_{n}$ from the SGZ stations. If, however, no records are close enough to SGZ, then obtaining F8 (and most of the other parameters as well) will be quite difficult. Finding $v_{3}$ on the velocity record can be difficult; we usually look for a change in slope somewhere before $v_{a}$. In Fig. $9 a$ (Event $A$ ) $v_{3}$ is slightly less than $3 \mathrm{~m} / \mathrm{s}$.

The geometric mean is used for the parameters $F 1$ through $F 7$ because the quantities they estimate are ratios, i.e., $F 1=a_{3} / g$. The geometric mean is the only measure of central tendency that is invariant under inversion of the data, i.e., we could have used $\mathrm{F} 1=\mathrm{g} / \mathrm{a}_{3}$. Our experience suggests that a reasonable range of values for the ratio parameters is 0.2 to 1.5 ; one should be wary of values larger than this. 


\section{Section 5. Atmospheric Acoustic Pulses}

The principal purpose of an analytic model of the surface ground motion from a UGT is to estimate the acoustic signal that is launched into the atmosphere as a result. These signals are calculated by the approximation of Huyghens-Kirchhoff integration over the moving ground surface. This surface is considered as a distributed source of mutually interfering acoustic wavelets. The theory behind this calculation is well-known. ${ }^{\text {in }}$ The response (i.e., the acoustic fluid velocity signal) at a point in the atmosphere directly above a UGT can be calculated from the following retarded integral over the ground surface:

$v(t)=\left.\left.\frac{1}{4 \pi}\right|_{11} ^{2 \pi}\right|_{i 1} ^{k}\left(\frac{1+\cos \theta}{a_{10} R} a_{n}\left(t-\frac{R}{a_{0}}\right)+\frac{\cos \theta}{R^{2}} v_{n}\left(t-\frac{R}{a_{10}}\right)\right] \operatorname{rdrd} \theta$

where $R$ is the slant distance from the observation point to a point on the moving ground surface, $a_{6}$ is the atmospt.. ric speed of sound (VA in our modelling parameter set), $a_{n}$ and $v_{n}$ are the vertical acceleration and relucity functions of our modelling, and rdrd $\Theta$ is an element of the ground surface area. The limit of integration over the moving ground is the radius $R_{c}$, corresponding to our $R C M \wedge X$, and $\theta$ is the angle the slant vector $R$ makes with the vertical. In this calculation we assume the atmosphere is uniform, i.e, $a_{1}$, is constant.

In this section we show several examples of the acoustic fluid velocity pulse resulting from our analytic parameterization of the surface ground motions. We also show how changes in some of the parameters affect the shape of this acoustic pulse profile. In all these examples, except as noted, the ubservation point is located $10 \mathrm{~km}$ above the ground and $1 \mathrm{~km}$ away from the vertical passing through SGZ, and $a_{1}$ is taken to be a mean value of $324 \mathrm{~m} / \mathrm{s}$ over this altitude range.

Figures 18a-c show acoustic pulses resulting from different fits to the surface ground motions for Event A. using the Warshaw model; Fig. 18d shows a puise using the Bernreuter model.

Only F3, F4, and F5 were changed in going from Fig. 18a to $18 \mathrm{~b}$. This had a substantial effect on the latter part of the pressure pulse. In going from Fig. 18 b to $18 \mathrm{c}$ RCMAX, VPOS, FV, XPV, VNEG, FW, XPW, 12, and F7 were changed. Again there was a substantial effect on the pulse. The parameter values for Fig. $18 \mathrm{c}$ are the final values. Figures $13 \mathrm{c}-\mathrm{d}$ allow us to compare the effect on the pressure pulse of the two different sets of radial functions for the velocity and acceleration extrema discussed in Section 2. As is cvident, the acoustic differences are not very great.

Figures $19 \mathrm{a}-\mathrm{b}$ show that the effects of differences hetween the radial models are not necessarily sn small. The pulse in Fig. 19a was calculated using Warshaw's, whereas the pulse in Fig. 19b was figured using Bernreliter's. All the other parameters are the same. These pulses are $8.75 \mathrm{~km}$ above the ground surface.

Figure 20 is the fluid velocity pulse for the parameters from Event $B$. Its shape is quite similar to that of Event A (Fig. 18c); from this we can conclude that, from an acoustic point of view, the differences in the ground motion between Events $A$ and $B$ are not that great. 

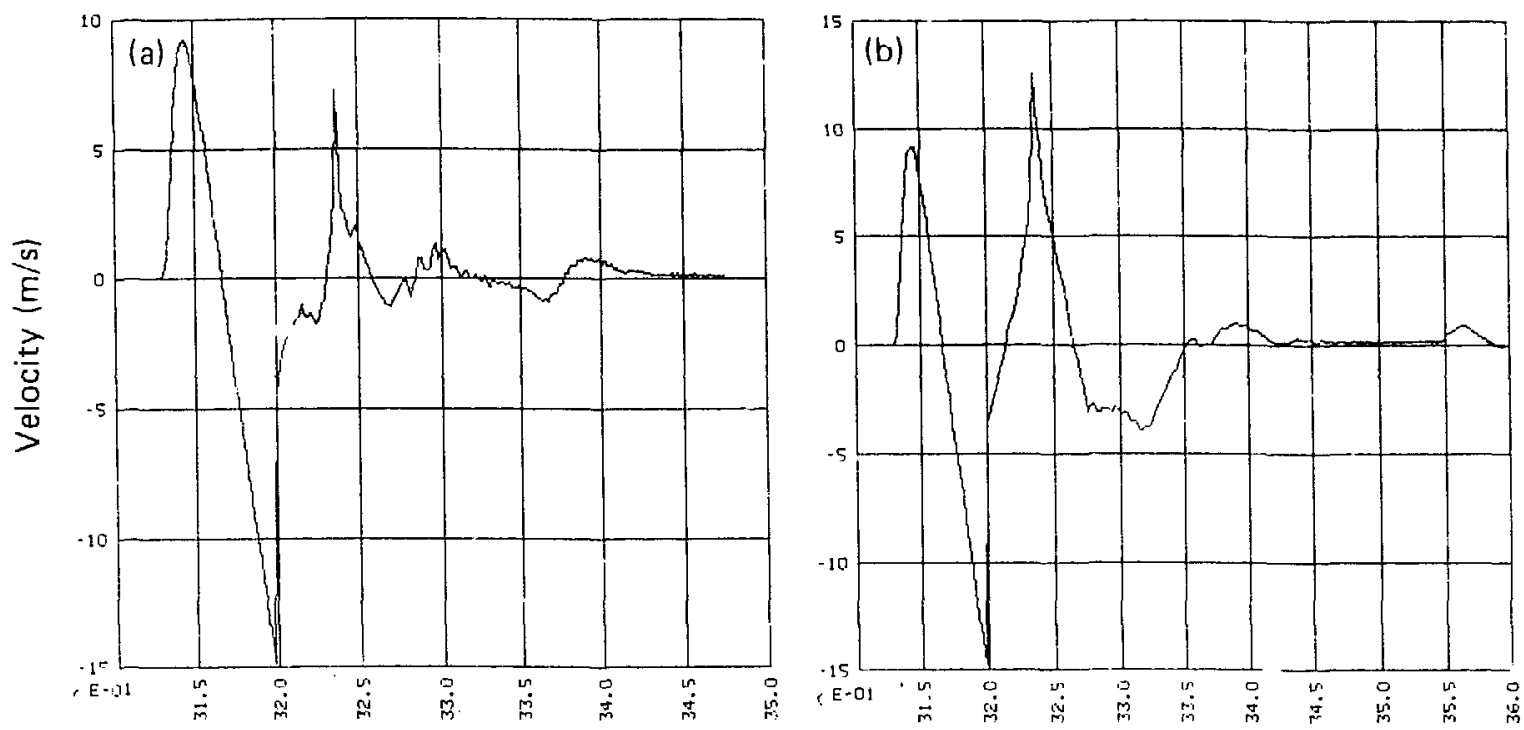

Time $(s)$

(a)

Parameters

$\begin{array}{rlll}\text { RCMAX } & =3200.0 & F T & =5.48 \\ \text { VA } & =324.0 & \text { XPT } & =1.57 \\ \text { VB } & =1735.0 & F 1 & =0.85 \\ \text { ZB } & =640.0 & F 2 & =0.50 \\ \text { APOS } & =47.40 & F 3 & =0.99 \\ \text { FA } & =0.32 & F 4 & =0.13 \\ \text { XPA } & =9.24 & \text { F5 } & =1.44 \\ \text { ANEG } & =235.20 & \text { F6 } & =0.16 \\ \text { FB } & =0.42 & \text { F7 } & =1.00 \\ \text { XPB } & =6.16 & \text { F8 } & =0.66 \\ \text { VPOS } & =4.44 & \text { Y } & =1.0 \\ \text { FV } & =0.85 & \text { ACON } & =2.61 E+05 \\ \text { XPV } & =1.26 & \text { AEXP } & =1.29 \\ \text { VNEG } & =-7.35 & \text { ARAT } & =3.75 \\ \text { FW } & =1.96 & \text { VCON } & =8.17 E+04 \\ \text { XPW } & =0.79 & \text { VEXP } & =1.53 \\ \text { DTSit } & =0.500 & \text { VRAT } & =1.31\end{array}$

(b)

Parameters

$\begin{array}{rlll}\text { RCMAX } & =3200.0 & \mathrm{FT} & =5.48 \\ \text { VA } & =324.0 & \text { XPT } & =1.57 \\ \text { VB } & =1735.0 & \mathrm{~F} 1 & =0.85 \\ \text { ZB } & =640.0 & \mathrm{~F} 2 & =0.50 \\ \text { APOS } & =47.40 & \mathrm{~F} 3 & =0.65 \\ \text { FA } & =0.32 & \mathrm{~F} 4 & =0.41 \\ \text { XPA } & =9.24 & \mathrm{~F} 5 & =0.67 \\ \text { ANEG } & =235.20 & \mathrm{~F} 6 & =0.16 \\ \text { FB } & =0.42 & \mathrm{~F} 7 & =1.00 \\ \text { XPB } & =6.16 & \text { F8 } & =0.66 \\ \text { VPOS } & =4.44 & \text { Y } & =1.0 \\ \text { FV } & =0.85 & \text { ACON } & =2.61 \mathrm{E}+05 \\ \text { XPV } & =1.26 & \text { AEXP } & =1.29 \\ \text { VNEG } & =-7.35 & \text { ARAT } & =3.75 \\ \text { FW } & =1.96 & \text { VCON } & =8.17 \mathrm{E}+04 \\ \text { XPW } & =0.79 & \text { VEXP } & =1.53 \\ \text { DTSH } & =0.500 & \text { VRAT } & =1.31\end{array}$

Figure 18. Acoustic pulses for Event A, calculated at a point $10 \mathrm{~km}$ above and $\mathbf{1} \mathrm{km}$ away from SGZ. (a) and (b) show pulses using the Warshaw radial model and preliminary parameter values, for comparison with (c) the final values using the Warshaw radial model, and (d) the final pulse using the Bern reuter radial model. 

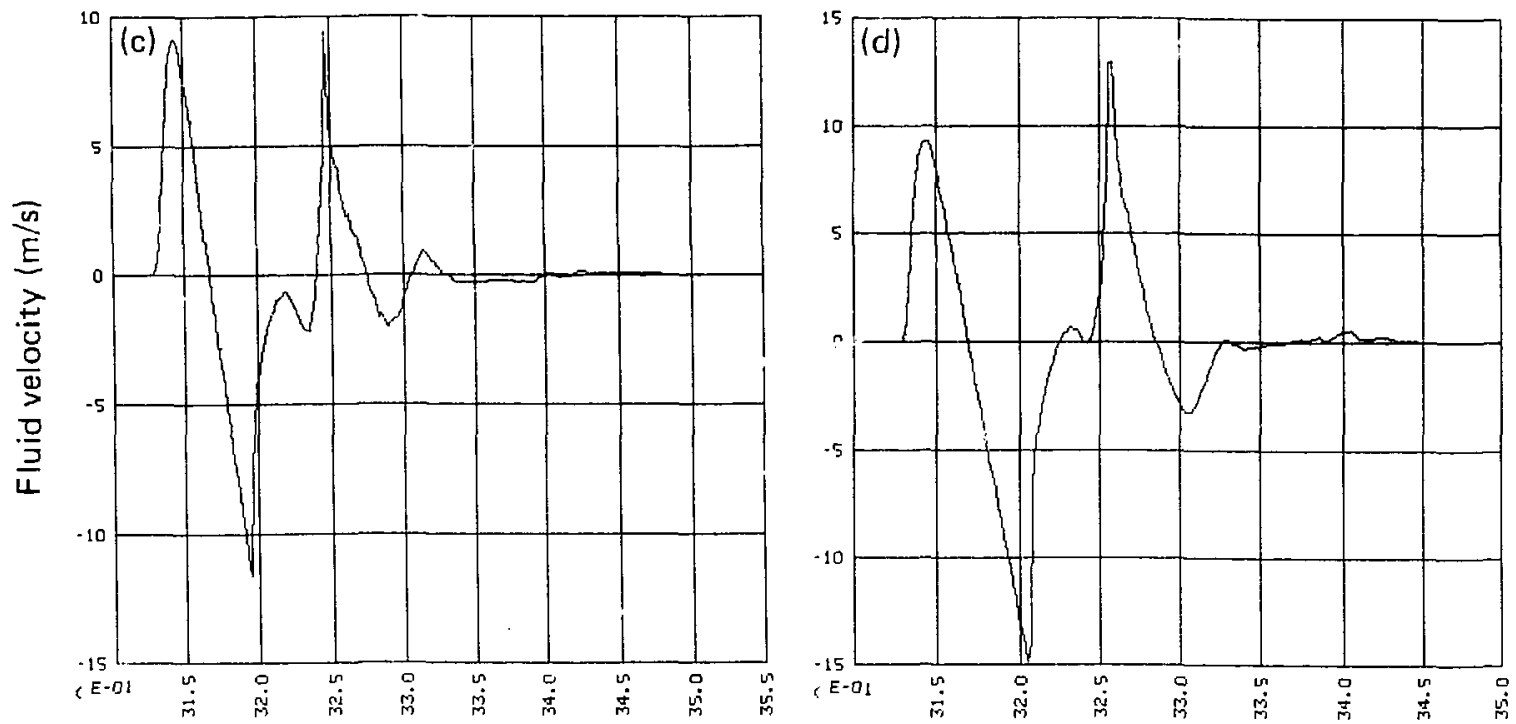

Time (s)

Parameters

$\begin{array}{rlr}\text { RCMAX } & =2300.0 \\ \text { VA } & =324.0 \\ \text { VB } & =1735.0 \\ \text { ZB } & =640.0 \\ \text { APOS } & =47.40 \\ \text { FA } & =0.32 \\ \text { XPA } & =9.24 \\ \text { ANEG } & =235.20 \\ \text { FB } & =0.42 \\ \text { XPB } & =6.16 \\ \text { VPOS } & =4.60\end{array}$

$\begin{array}{ll}\text { FV } & =1.00 \\ \text { XPV } & =1.46 \\ \text { VNEG } & =-7.39 \\ \text { FW } & =1.45 \\ \text { XPW } & =1.66 \\ \text { DTSH } & =0.500 \\ \text { FT } & =5.48 \\ \text { XPT } & =1.57 \\ \text { F1 } & =0.85 \\ \text { F2 } & =0.75 \\ \text { F3 } & =0.65\end{array}$

$\mathrm{F} 4=0.14$

$\mathrm{F5}=0.67$

$F 6=0.16$

$\mathrm{F7}=0.85$

$\mathrm{F8}=0.66$

$Y=1.0$

$A C O N=2.61 \mathrm{E}+05$

$A E X P=1.29$

ARAT $=3.75$

VCON $=8.17 \mathrm{E}+04$

VEXP $=1.53$

VRAT $=1.31$

Fig. 18 continued. 

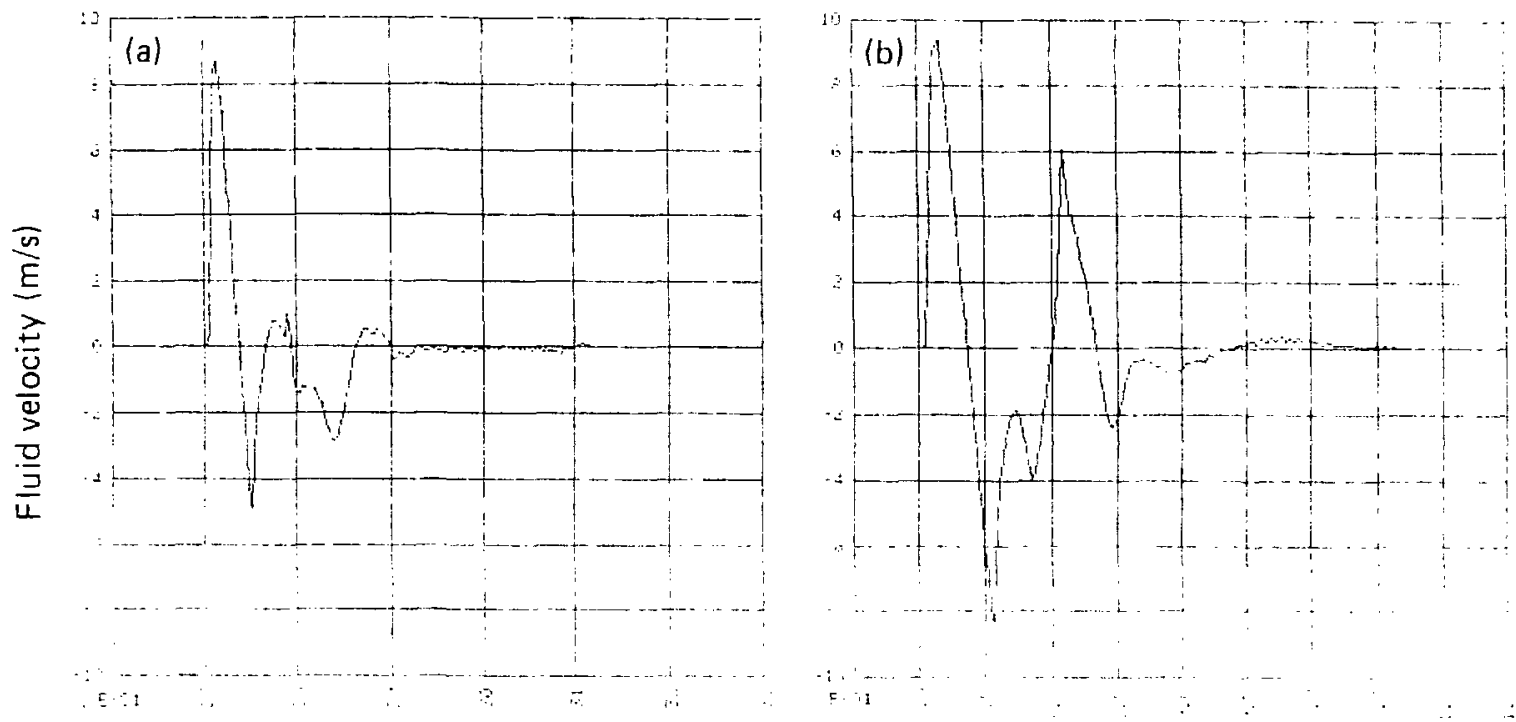

Time (s)

Parameters

$\begin{array}{llll}\text { RCMAX } & =2800.0 & F T & =6.05 \\ V A & =329.0 & \text { XPT } & =1.11 \\ \text { VB } & =1565.0 & F 1 & =2.36 \\ \text { ZB } & =565.0 & F 2 & =0.89 \\ \text { APOS } & =146.90 & F 3 & =0.35 \\ \text { IA } & =1.70 & F 4 & =0.44 \\ \text { XPA } & =1.48 & F 5 & =0.75 \\ \text { ANEG } & =205.80 & F 6 & =0.25 \\ \text { F8 } & =4.76 & F 7 & =0.90 \\ \text { XPB } & =0.91 & F 8 & =0.55 \\ \text { VPOS } & =6.27 & Y & =1.0 \\ \text { FV } & =1.66 & \text { ACON } & =2.84 E+08 \\ \text { XPV } & =1.09 & \text { AEXP } & =2.33 \\ \text { VNEG } & =-7.21 & \text { ARAT } & =1.68 \\ \text { FW } & =3.90 & \text { VCON } & =1.05 E+07 \\ \text { KPW } & =1.56 & \text { VEXP } & =2.28 \\ \text { DTSH } & =0.300 & \text { VRAT } & =0.79\end{array}$

Figure 19. Comparison of acoustic pulses created with the Warshaw and Bernreuter radial models. These pulses were calculated at a point $8.75 \mathrm{~km}$ above and $1 \mathrm{~km}$ away from the vertical through SGZ. (a) uses the Warshaw radial model, while (b) uses the Bern reuter radial model. 


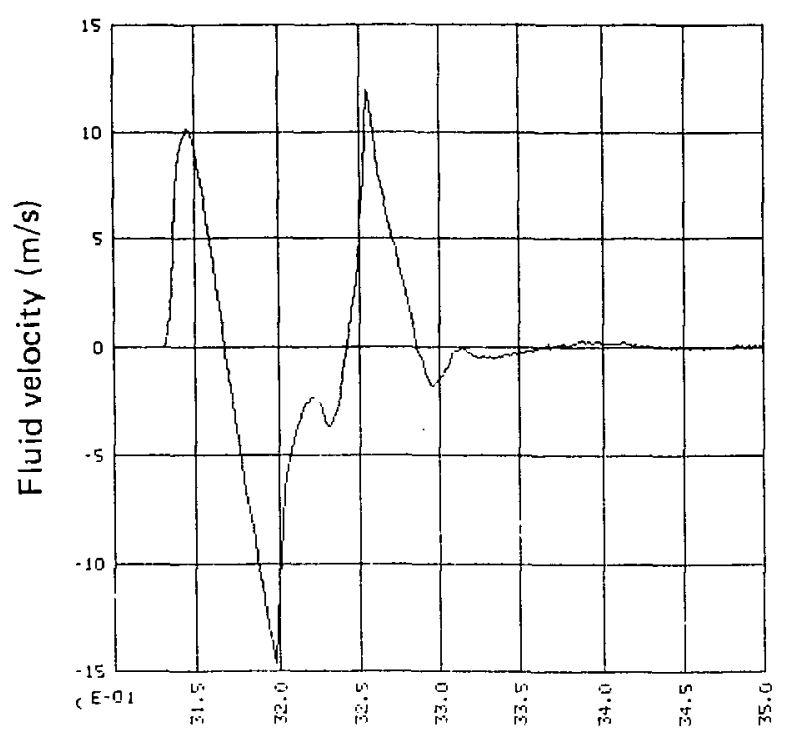

\begin{tabular}{|c|c|c|c|}
\hline \multicolumn{4}{|c|}{ Parameters } \\
\hline \multicolumn{2}{|c|}{ RCMAX $=3500.0$} & DTSH $=$ & 0.410 \\
\hline$V A=3$ & 324.0 & $F T=$ & 4.99 \\
\hline$V B=1$ & 1843.0 & $X P T=$ & 1.32 \\
\hline$Z B=7$ & 701.0 & $F_{1}=$ & 1.13 \\
\hline APOS= & 62.16 & $F 2=$ & 0.75 \\
\hline$F A=$ & 0.53 & $F 3=$ & 0.58 \\
\hline$X P A=$ & 4.09 & $F_{4}=$ & 0.34 \\
\hline ANEG $=$ & $=193.66$ & $\mathrm{~F} E=$ & 0.65 \\
\hline $\mathrm{FB}=$ & 1.15 & $F G=$ & 0.29 \\
\hline $\mathrm{XPB}=$ & 2.19 & $F 7=$ & 0.92 \\
\hline UPOS $=$ & 4.22 & $\mathrm{Fg}=$ & 0.44 \\
\hline$F U=$ & 0.91 & & \\
\hline YPV $=$ & 1.91 & & \\
\hline UNEG $=$ & -6.40 & & \\
\hline$F_{N} N=$ & 1.01 & & \\
\hline$X P|k|=$ & 2.18 & & \\
\hline
\end{tabular}

Time (s)

Figure 20. Acoustic pulse for Event $B$ using the Warshaw radial model and the final set of parameters shown in Table 6. This pulse was calculated at a point $10 \mathrm{~km}$ above and $1 \mathrm{~km}$ away from the vertical through SGZ.

\section{Section 6. Data Used for the Model}

We treated all the data as received, '.e., without modification.

Figures $21 \mathrm{a}-\mathrm{b}$ are two examples of actual accelerometer records. They illustrate some of the aspects of the data (compare with Fig. 17). Most of the data was taken by Los Alamos National Laboratory. In general, the format and presentation of this data is quite useful for our purposes. The time scales of the plots are particularly appropriate for our needs.

We have experienced three sources of difficulty with this data. The first is that the slapdown acceleration peaks are frequently clipped. The problems this causes were discussed in Section 2.1.

Second, noise in the acceleration records often distorts the velocity records. This happens because the velocity records we have been using are not measurements in the true sense of the word but are obtained by integrating the acceleration records. As we mentioned in Section 2.2, this primarily affects the data during and after slapdown. However, the data sometimes appears to be distorted prior to slapdown.

Third, the accelerometer coverage on various explosive events ranges from very sparse to barely adequate for our needs. The largest data set we have for one event contains 14 surface acceleration records, some of which are only partially useful. Many events have data sets with no records beyond the presumed extent of spall, which is a troublesome omission if one wishes to calculate atmospheric pressure pulses. Figure 10 shows an example of a problem this limited coverage can cause.

In order to make a crude estimate of how many accelerometers might be necessary to thoroughly cover the ground surface, we would like to determine how much surface area a single accelerometer can adequately represent. We have found that accelerometer records taken less than $200 \mathrm{~m}$ apart can be significantly different, but this distance probably represents an extreme; a more typical distance might be $400 \mathrm{~m}$. Let us therefore assume that a single accelerometer adequately covers the area within a radius of $200 \mathrm{~m}$ about itself. It follows that, within a radius of $1000 \mathrm{~m}$ about SGZ, at least 25 accelerometers are needed to cover the entire surface:

$\mathrm{n}=\pi \mathrm{r}_{1}^{2} / \pi \mathrm{r}_{2}^{2}=\left(\mathrm{r}_{1} / \mathrm{r}_{2}\right)^{2}=(1000 / 200)^{2}=25$ 
(a)
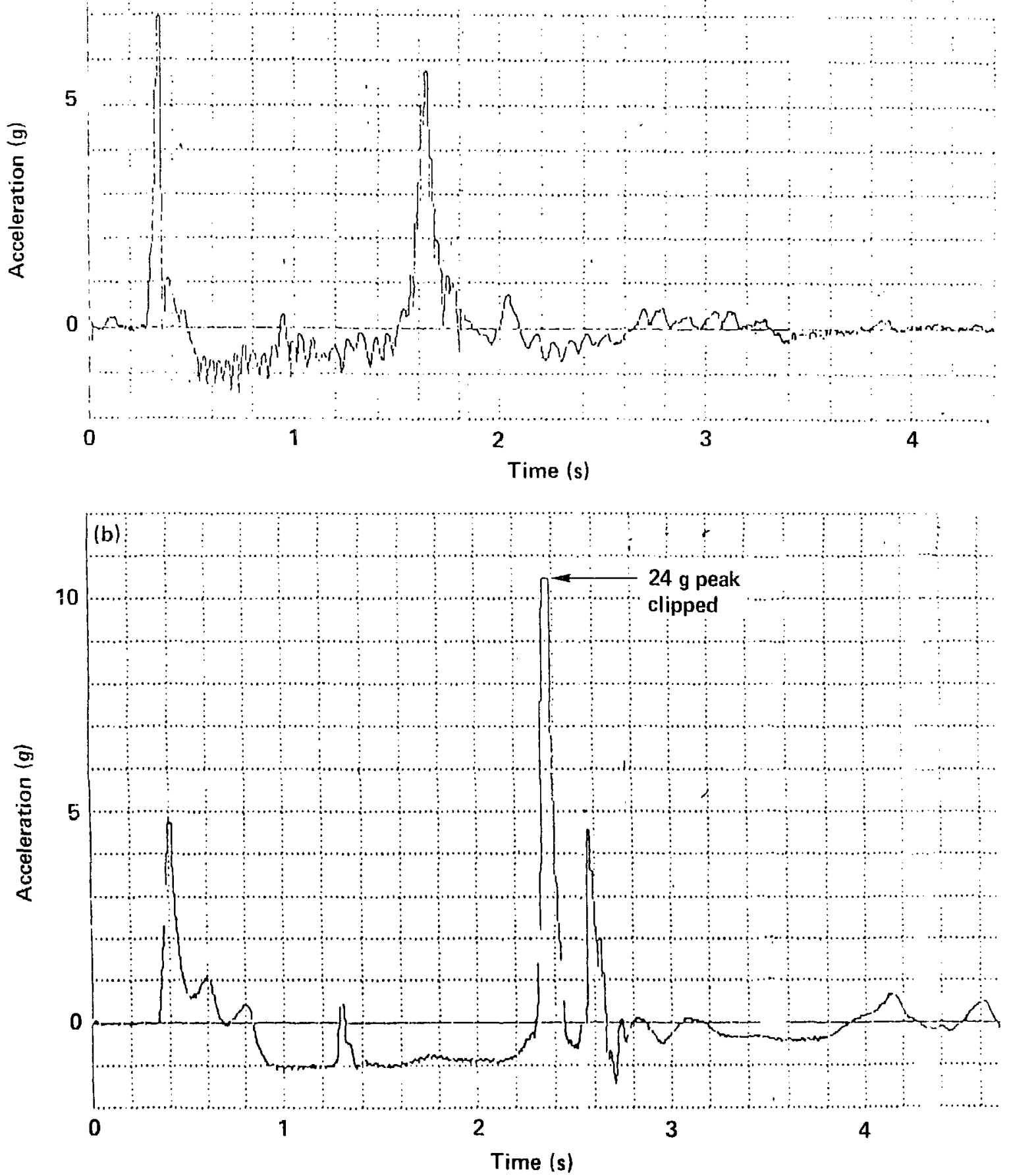

Figure 21. Accelerometer records taken at SGZ. These records show some of the problems we often encounter, such as (a) noise and (b) clipping, as well as details that our model does not fully account for. 
If we suppose that accelerometers more than $1000 \mathrm{~m}$ from SGZ are good for coverage within a radius of $500 \mathrm{~m}$ about themselves, then another 32 accelerometers are needed to cover the surface between 1000 and $3000 \mathrm{~m}$. We believe that any study of the ground motion attempting to take into account the fact the ground motion is azimuthally variable will require at least this degree of coverage.

\section{Section 7. Critique of the Present Model}

This model is an initial guess at a time profile and radial dependence of what are hoped to be the most relevant gross features of UGT induced ground surface motions.

The major problems in the time profile are in the shoulder $a_{3}$ and $a_{4}$ and the reverberations $a_{4}, a_{4}$, and $a_{11}$. As indicated in Section 4 and Fig. 17, the model for the shoulder cannot encompass the wide variety of acceleration profiles seen here. However, it does a reasonable job of approximating them.

The reverberations after slapdown are more of a problem. Figure $21 \mathrm{~b}$ is a good example of an acceleration record that does not look remotely like our model after the slapdown peak. For future woik, jt might be appropriate to have some sort of parameterization that allows $a_{x}<0, a_{4}>0$ and so on, or that allows fewer or more acceleration peaks after slapdown.

In terms of the radial dependence, the variation in the data for $a_{2}$ (in many cases) and for $a_{-}$(in most cases) is so great that no monotone function will fit then; very well. The vartation is probably due to the fact that the ground motion is not cylindrically symmetric; if this is so, then any cylindrically symmetric model will have this problem. An asymmetric model might use an azimuthal modulation of the existing radial modelling.

It has turned out to be more effective for parameterizing this model to estimate F2 through F5 using the widths and magnitudes of $v_{i}$ and $v_{i}$, even though F2 and F3 actually specify accelerations. If we had been able to anticipate this it might have been better to have the parameters specify the widths of $v_{n}$ and $v$, directly.

The parameterization of $v_{3}$ is somewhat unsatisfactory because there are parameter sets for which $v_{3}>v_{4}$, and thus $t_{3}>t_{4}$ if $R C$ is larger than some critical value. This critical value depends on several of the other parameters in a complex fashion. An ideal model would have simple constraints on each parameter, independent of the others, such that whenever the constraints are met, basic properties of the model (such as $t_{4}>t_{3}$ ) are not violated. This parameterization of $v_{3}$ does not have this property. We have solved the problem by adjusting $v_{3}$ to be slightly less than $v_{4}$ whenever the critical radius is exceeded.

\section{Section 8. Overview of the Modelling Process}

This section provides general comments that do not depend on the specific model and parameterization used.

First, it is clear that any complete model for UGT induced ground motion must include a time profile for the ground motion and a radial dependence of some sort. Our approach has been to estimate the radial dependencies of specific points on the profile independently of their effect on the profile's shape and then evaluate the fit of the overall profile. The azimuthal dependence has been ignored or averaged out.

The estimation of the radial dependencies is a problem best handled by conventional statistical methods. The methods are straightforward: depending on the radial function, some sort of least squares 
method is appropriate. Subtleties arise when a constraint of the sort discussed in Sectivis 1 and 7.1 causes local minima, or when we try to take into account the fact that the data do not have homogeneoun variances.

Difficulties arise when there is insufficient data (see Fig. 20); then the investigator's arbitrary choice is required. This in turn leads to radial fits that are not best in any statistical sense and opens up the possibility that different investigators could obtain substantially different predictions based on the same data.

We have not attempted to evaluate the :s. of the overall time profiles by statistical methods because evaluating the error of the fit would require extremely clean data. Miscellaneous noise and calibration errors in the data would inflate the estimate of the error of the fit. Also, some parts of the time profile are obviously more important than others, and we don't know enough yet to determine appropriate statistical weightings.

As a consequence, our evaluation of the overall profile fits is basically visual. We look at the entire set of superimposed plots, eliminate as many gross errors as possible, and attempt to balance out the more subtle errors so that some are in one direction and some are in the other. The discussion in Section 3.2 gave some indication of this process.

If it were practical to evaluate the fit of the profile by statistical methods, it might be possible to take a general approach in which the radial dependencies were evaluated simultaneously with the profile fits. Then the radial dependencies might apply to the entire profile rather than to selected points on it.

\section{Section 9. Summary}

We have presented some details of a specific ground motion model (Section 1); two examples of its application, including some discussion of the choices involved in obtaining the radial parameters (Sections 2 and 3); and a separate discussion of our methods for choosing the ratio parameters (Section 4). Section 5 presented examples of the model's use in calculating the acoustic air pressure pulses resulting from the ground motion; Section 6 discussed the quality of the data we worked with; Section 7 critiqued our model; and Section 8 discussed general aspects of our approach to fitting the model.

The specific model is only a beginning attempt at modelling UGT induced ground motion in which spall is present. It is capable of dealing with a wide variety of radial dependencies and can lead to a substantial variely of air pressure pulses. Despite its sometimes severe limitations and gross uver. simplifications, we believe it has done a surprisingly good job of reproducing several sets of ground motion data.

\section{Acknowledgments}

A note of acknowledgment is due to Robert Fitzhugh and Larry Barrick of the Los Alamos National Lahoratory for their willingness to discuss the data, help us to understand its problems, and guide us in appropriate modifications.

We are grateful to LLNL's Vern Wheeler for providing us with the bulk of our data and Eugene Jackson for aiding us in the interpretation of the surface ground motion records.

A special note of thanks is due to Steve Warshaw of LLNL for his substantial contributions to both the work and report, particularly for his contributions to Section 5. 


\section{References}

1. F. M. Sauer, Nuclear Geoplosics, A Sourcebook of Underground Phenomena and Effects of Nuclear Explosions. Part Four-Empirical Analysis of Ground Motion and Cratering, Defence Atomic Support Agency, Washington, D.C., DASA-1285 (IV) (1964), p. 29.

2. D. L. Bernreuter, E. C. Jackson, and A. B. Miller, Control of the Dynamic Environment Produced by Underground Nuclear Explosives, Lawrence Livermore National Laboratory, Livermore, CA, UCRL-72122 (1969).

3. NAG Fortran Library Manual Mark 6, E04GAF, Numerical Algorithms Group Incorporated, Downers Grove, IL (1977).

4. J. D. Eisler and F. Chilton, "Spalling of the Earth's Surface by Underground Nuclear Explosives," fournal of Geophysical Research, 69 (24) (1964).

5. J. D. Jackson, Classical Elictrudynamics (John Wiley \& Sons, New York, 1975).

6. J. R. Banister, A Program for Predicting Ground Motion Induced Air Pressures, Sandia Laboratories, Albuquerque, NM, SAND78-2361 (1979). 


\section{Appendix}

This appendix contains formulas for all of the points $t_{1}$ through $t_{11}, t_{11}, t_{1,}, t_{1}, t_{1,}, v_{1}$ through $v_{11}, v_{1,}, v_{3,}$, $v_{Y,} v_{i}$, and $a_{1}$ through $a_{11}$, which are used in our work.

The following symbol definitions are used:

$g$ is the acceleration of gravity $\left(9.8 \mathrm{~m} / \mathrm{s}^{2}\right)$,

$Z B$ is the depth of burial,

$V B$ is the average speed of propagation of the shockwave from the point of explosion to the surface,

$\mathrm{RC}$ is the surface distance from $\mathrm{SGZ}$ to a ground motion recording location. The symbols $\mathrm{F} 1$ through F8, APOS, FA, XPA, ANEG, FB, XPB, VPOS, FV, XPV, VNEG, FW, XPW, DTSH, FT, and XPT are parameters to be estimated.

Except for $a_{10}, v_{r}$, and $v_{n}$, all of the following points are expressed solely in terms of the above parameters:

$$
\begin{aligned}
& t_{2}=\frac{1}{V B}, R C^{2}+Z^{2} \\
& a_{1}=a_{11}=0 \\
& \mathrm{a}_{2}=\frac{\mathrm{APOS}}{1+\left(\mathrm{FA} \frac{\mathrm{RC}}{\mathrm{ZB}}\right)^{\mathrm{TPA}}} \\
& \mathrm{a}_{3}=\mathrm{a}_{4}=(\mathrm{Fl}) \mathrm{g} \\
& a_{5}=a_{b}=(-F 7) g \\
& a_{-}=\frac{\text { ANEG }}{1+\left(\mathrm{FB} \frac{\mathrm{RC}}{\mathrm{ZB}}\right)^{\mathrm{WB}}} \\
& a_{8}=(F 2) g \\
& \mathbf{a}_{9}=(-\mathrm{F} 2) \mathrm{g} \\
& v_{.1}=\frac{\text { VPOS }}{1+\left(\mathrm{FV} \frac{\mathrm{RC}}{\mathrm{ZB}}\right)^{1 \Gamma \mathrm{V}}} \\
& \mathrm{a}_{10}=(\mathrm{F} 3) \mathrm{a}_{\mathrm{s}} \\
& v_{1}=v_{8}=v_{9}=v_{11}=0 \\
& v_{i}=(F 4) v_{11} \\
& v_{i i}=\frac{V N E G}{1+\left(F W \frac{R C}{Z B}\right)^{\mathrm{VWW}}} \\
& \mathrm{v}_{\delta}=(-\mathrm{F} 5) \mathrm{v}_{\hat{i}} \\
& \Delta t=t_{5}-t_{4}=\frac{\text { DTSH }}{1+\left(F T \frac{R C}{Z B}\right)^{x \Gamma T}} \\
& v_{3}=v_{\mathrm{u}}\left\{1-(1-\mathrm{F} 8)\left[1+\left(\mathrm{FT} \frac{\mathrm{RC}}{\mathrm{ZB}}\right)^{\mathrm{x} \mathrm{T}}\right]^{-1}\right\}
\end{aligned}
$$

The remaining points are expressed in terms of the parameters, the above points, and each other.

$$
\begin{array}{ll}
t_{1}=t_{2}-\frac{2 v_{3}}{a_{3}+2 a_{2}} & t_{5}=t_{4}+\Delta t \\
t_{3}=t_{2}+\frac{2 v_{3}}{a_{3}+2 a_{2}} & t_{6}=t_{5}+\frac{v_{6}-v_{5}}{a_{5}} \\
t_{4}=t_{3}+\frac{v_{4}-v_{3}}{a_{3}} & t_{6}=\frac{a_{7} t_{6}-a_{6} t_{7}}{a_{7}-a_{6}} \\
t_{41}=\frac{a_{4} t_{5}-a_{5} t_{4}}{a_{4}-a_{5}} & t_{7}=t_{6}+F 6\left(t_{2}-t_{1}\right)
\end{array}
$$




$$
\begin{aligned}
& \mathrm{t}_{\mathrm{s}}=\mathrm{t}_{7}-\frac{2 \mathrm{v}_{7}}{\mathrm{a}_{\mathrm{x}}+\mathrm{a}_{7}} \\
& v_{2}=\frac{a_{2} v_{3}}{a_{3}+2 a_{2}} \\
& t_{7}=\frac{t_{8}+t_{9}}{2} \\
& v_{4}=v_{11}-\frac{1}{2} a_{4}^{2} \frac{s t}{a_{4}-a_{5}} \\
& \mathrm{t}_{4}=\mathrm{t}_{\mathrm{s}}+4 \frac{\mathrm{v}_{\mathrm{i}}}{\mathrm{a}_{\mathrm{s}}} \\
& v_{5}=v_{4}+\frac{1}{2}\left(a_{4}+a_{5}\right) \Delta t \\
& \mathrm{t}_{i \mathrm{i}}=\mathrm{t}_{4}+2 \frac{\mathrm{v}_{i}}{\mathrm{a}_{9}} \\
& v_{b}=v_{a}-\frac{1}{2} a_{6}^{2} \frac{t_{7}-t_{b}}{a_{6}-a_{7}} \\
& t_{10}=t_{9}+F 3 \frac{2 v_{i}}{a_{4}} \\
& v_{7}=v_{6}+\frac{1}{2}\left(a_{6}+a_{7}\right)\left(t_{7}-t_{6}\right) \\
& t_{11}=t_{11}-2 \frac{v_{11}}{a_{111}} \\
& v_{10}=v_{i j}+\frac{1}{2} a_{11}\left(t_{10}-t_{i i}\right)
\end{aligned}
$$

The generic relationship is that for any pair of adjacent points,

$v_{1,1}-v_{1}=\frac{1}{2}\left(a_{1+1}+a_{i}\right)\left(t_{1}+1-t_{1}\right)$.

It should be noted that if $v_{4}<v_{3}$ then $t_{4}<t_{3}$, and the model should not allow this. Since it is possible to produce sets of parameters that cause this but are otherwise acceptable, there should s some sort of check for this condition in any code that calculates these values In our computes ades, we set $v_{3}=v_{4}-10^{4}$ whenever $\left(v_{4}-v_{3}\right)<10^{4}$. In addition, the above definitions require $F 3<1$, and $F 8<1$. Except for VNEG, all parameters are positive. Note that the parameter RCMAX, discussed in the text, does not appear here. RCMAX is not necessary for the calculation of the ground motion from a set of the above parameters, but it is very important for the estimation of the parameters. It is also essential for the calculation of the atmospheric acoustic pulse. 